\title{
Vortex-induced interfacial waves in liquid-liquid flows across cylindrical bluff bodies of various sizes
}

\author{
Mohd Izzudin Izzat Zainal Abidin ${ }^{\mathrm{a}, \mathrm{b}}$, Kyeong Hyeon Park ${ }^{\mathrm{a}}$, Panagiota Angeli ${ }^{\mathrm{a} *}$ \\ aThAMeS Multiphase, Department of Chemical Engineering, University College \\ London, London WC1E 7E, UK \\ ${ }^{b}$ Department of Chemical Engineering, Faculty of Engineering, University of \\ Malaya, Kuala Lumpur 50603, Malaysia
}

\begin{abstract}
$\underline{\text { ABSTRACT }}$
The use of transverse cylinders of various sizes for generating vortex-induced waves in stratified oil-water pipe flows is investigated in this work. From CFD simulations in single phase flows, cylinders with $2 \mathrm{~mm}$ and $8 \mathrm{~mm}$ in diameter were selected which are found to generate vortices with frequencies in the range of 1 to $100 \mathrm{~Hz}$. The experimental work was conducted in a $8 \mathrm{~m}$ long acrylic test section with $37 \mathrm{~mm}$ internal diameter. High speed imaging was used to study the flow patterns and the interfacial wave characteristics while velocity fields and the characteristics of the vortices shed by the cylinders in the water phase were obtained with Particle Image Velocimetry. It was found that the vortex properties generated by different cylinder sizes are reflected on the interfacial wave characteristics. The frequencies of both the waves and the vortices agreed for all cases studied, while the wave amplitudes increased with increasing cylinder diameter. The Strouhal number for the $2 \mathrm{~mm}$ cylinder had the same value of 0.20 as in unbounded flows, while for the $8 \mathrm{~mm}$ cylinder and small distance from he wall, the Strouhal number was equal to 0.27 . The presence of the transverse cylinders modified the two-phase flow pattern maps and reduced the transition from stratified to dispersed flows to lower mixture velocities compared to flows without cylinders. The transition boundaries were shifted further towards lower mixture velocity with increasing cylinder diameter.
\end{abstract}

Keywords: Oil-water flows; Vortex-induced waves; High speed imaging; Strouhal number; Flow pattern transition.

*Corresponding author: p.angeli@ucl.ac.uk 


\subsection{Introduction}

The simultaneous flow of two immiscible liquids in a pipe is commonly encountered in the chemical and petroleum industries. Different geometrical distributions of the two phases, also called flow patterns or regimes, are established depending on the flow conditions, inlet geometry and pipe size, which influence essential system properties such as pressure gradient and hold-up. Therefore, knowledge of the flow patterns and their transition boundaries becomes important for accurate design of equipment and development of predictive models. Numerous studies have been conducted on oil-water pipe flow to identify and characterize the flow regimes at various flow conditions [13]. The flow pattern transitions, in particular from stratified to non-stratified flows, have attracted a lot of interest. Stratified flow is commonly observed at relatively low flow velocities where the gravitational force dominates over the inertial and viscous forces. In this regime, both phases retain their continuity, forming two parallel layers separated by a clear interface. Transition to non-stratified flows is identified with the onset of drop entrainment from interfacial waves of one phase into the other. The interfacial waves in stratified flows grow in amplitude due to the Kelvin-Helmholtz instability as they propagate along the pipe until they reach a critical amplitude necessary for drops to detach $[5,6]$. The mechanism of transition, however, is yet to be fully understood due to lack of experimental data near the transition boundaries, as experimentally it is very difficult to localize the droplet detachment events and to investigate the characteristics of breaking waves along the pipe. To facilitate the studies near the transition region in oil-water flows, a novel approach was developed in recent works by Park et al. [6] and Chinaud et al. [7] in which a bluff body of a cylindrical shape located in the water phase, transverse to the flow direction, was used to actuate interfacial waves and to localize the onset of drop detachment. Park et al., studied the characteristics of the generated interfacial waves using high speed imaging and showed the effect of bluff body on the transition boundary between stratified and dispersed flows. Further studies on the same system by Chinaud et al. with high-speed PIV showed that the frequency of the generated interfacial waves matched the frequency of the von Karman vortices shed by the bluff body. Based on these works, the effects of different bluff bodies on the vortex and interfacial wave characteristics are studied here. 
Flow across a bluff body triggers different phenomena relative to obstacle-free flow even at low velocities, such as flow separation, vortex shedding and turbulence. Flow separation occurs above a critical Reynolds number, and results in shedding of alternate vortices, which cause a local pressure change each time a vortex is formed. The vortices are convected downstream as a vortex street, known as the von Karman vortex street. Various parameters have been investigated in the wake of the bluff body including vortex shedding frequency, lift and drag coefficients, wake velocity, length and width of the vortex formation region. Flows across different bluff body geometries have also been studied such as rectangular [8-10] and elliptical [11]; the cylinder, however, remains the most popular geometry due to its simplicity and wide range of industrial applications.

A detailed review of the vortex dynamics in the wake of the cylinder was given by Williamson [12] who also considered three-dimensional effects. Studies have focused on cylindrical structures in the sub-critical regime $\left(\operatorname{Re}_{D}=10^{2}\right.$ to $\left.10^{5}\right)$ where the vortex shedding is almost independent of the cylinder based Reynolds number, defined as $\operatorname{Re}_{D}$ $=\rho u D / \mu$ where $u$ is the fluid velocity, $\rho$ is the fluid density, $D$ is cylinder diameter and $\mu$ is the fluid viscosity. The periodic process of vortex shedding is a dominant feature of flows across bluff bodies and the shedding frequency, $f_{v}$ is linked to the Strouhal number, $S t$, which is defined as:

$$
S t=f_{v} D / u
$$

In the sub-critical regime, St remains almost constant at 0.20 . In many engineering applications, the flows across a bluff body are in the presence of a wall or a free surface in its proximity. The presence of confinement restricts the flow in the gap between the wall and the bluff body, resulting in more complex wake structures compared to those in unconfined flows. In this case, an additional parameter called gap ratio, $\gamma$, influences the wake dynamics. It is defined as the ratio of the distance from the bluff body to the wall, $h$, to the cylinder diameter;

$$
\gamma=h / D
$$


Based on numerical studies by Zovatto and Pedrizzetti [13] on flows past a cylinder between parallel walls, as the gap ratio is reduced, the resulting wake causes the vorticity at the boundary layer on the wall to rise and approach the wake. The vortices facing the wall may interact with the vorticity from the wall boundary layer of opposite sign and delay the transition from the steady to the periodic vortex shedding regime to higher Reynolds numbers compared to unconfined flows [10, 14]. Experimentally, it has been reported that vortex shedding is suppressed or extremely weak at very small gap ratios less than 0.30 [15-17].

When the flow across a bluff body is near a free surface, two additional dimensionless parameters are introduced, namely the submergence depth, $h_{s}$ defined as the distance from the surface to the bluff body, and the Froude number, $F r$, determined as:

$$
F r=u / \sqrt{g D}
$$

When the distance of the bluff body from the surface is small, interactions may occur between the free surface and the vortices. A two-dimensional numerical analysis conducted by Reichl et al. [18] for a flow past a cylinder near a free surface at two different ratios of submergence depth to cylinder diameter showed that at $F r=0$ and $h_{s} / D=0.55$, the resulting vortices are symmetric and resemble the case of a fully submerged cylinder. A small local surface distortion started to appear at $\mathrm{Fr}=0.3$ and the distortion increased at $F r=0.4$. A similar trend was reported by Lin \& Huang [19], where at high $F r$, larger surface distortions were observed. Sheridan et al. [20], based on experimental investigations, reported that vortices are attached to the free surface. The submergence depth influences the vortex shedding from the cylinder as the amount of fluid transported into the wake region is controlled by the gap between the top of the cylinder and the surface. The interface distortion causes asymmetry in the wake downstream, with the vortex street skewed to a downward direction, leading to variations in the cylinder lift coefficient. A change in the Strouhal number for different ratios of submergence depth to cylinder diameter was reported by Reichl et al. [18]. The value of $S t$ initially increased as the submergence depth reduced before declining to a very low value. This behaviour was related to the average velocity in the region above the cylinder. At low submergence depth, less fluid is transferred into the wake 
which reduces the shedding frequency and the Strouhal number. Many studies on the effects of the wall and free surface on the flow past a bluff body have also been conducted numerically with Reynolds Average Navier-Stokes (RANS) approaches for two-dimensional cases [21-27] and Large Eddy Simulations (LES) for threedimensional cases [28-30] as it is more convenient to vary the geometry and the physical properties compared to experiments.

This work investigates the characteristics of vortex-induced interfacial waves for different vortex shedding properties and their influence on the transition from separated to dispersed flows. For the first time the frequencies and properties of the vortices and of the interfacial waves are varied by using transverse cylinders of different diameters. The sizes of the cylinders are designed numerically. Experiments in two-phase oilwater flows were carried out to relate the properties of the vortices shed by the cylinders to the interfacial wave characteristics and to investigate the flow pattern transitions in the presence of the cylinders.

\subsection{Methodology}

2.1 Numerical analysis: Design and selection of cylinder diameters

Previous studies by Park et al. [6] showed that a $5 \mathrm{~mm}$ cylinder in a $37 \mathrm{~mm}$ ID test pipe generated vortices with frequencies ranging from 10 to $40 \mathrm{~Hz}$ in single phase flows for velocities in the range of 0.3 to $1.0 \mathrm{~m} / \mathrm{s}$. In the same system, it was also shown by Chinaud et al. [7] that the vortex shedding frequencies from a cylinder submerged in the water layer of an oil-water pipe flow matched those of single phase flow, while in the middle plane of the pipe the flow fields were comparable to two-dimensional flows. To extend the frequency range of the vortex shedding and observe its influence on the interfacial waves, in the current work, numerical simulations are performed for different cylinder diameters to identify the sizes that would deliver vortex shedding frequencies spanning two orders of magnitude, between 1 and $100 \mathrm{~Hz}$. Based on the previous findings and to reduce computational time, two-dimensional simulations are carried out for single water phase. For the simulations the commercial finite volume CFD code FLUENT was used which has been successfully applied previously for both two-dimensional and three-dimensional flows across bluff bodies by various investigators [21, 22, 28, 30-33] 
The single phase flow of water past a $5 \mathrm{~mm}$ cylinder was chosen as the base case of the numerical analysis considering the availability of experimental data from previous studies [7] for the purpose of comparison and validation. Simulations were performed on a plane located at the center of the pipe along the flow direction as illustrated in Figure 1. The length of the domain is $0.1 \mathrm{~m}$ upstream and $0.5 \mathrm{~m}$ downstream the cylinder respectively. The height of the flow domain is taken equal to the pipe diameter in the work of Chinaud et al. [7], which is $0.037 \mathrm{~m}$ and the cylinder diameter $D$ is equal to $5 \mathrm{~mm}$. The computational domain was meshed with quadrilateral cells with refinements in the vicinity of the cylinder and in the wake region, to capture the rapid changes of velocity in those areas due to the formation of the von Karman vortex street, which resulted in a total number of cells of $150 \mathrm{k}$. The turbulent $k$ - $\varepsilon$ model was used because of its robustness and reasonable accuracy with low computational cost compared to other turbulence models.

The simulation results of the flow past the $5 \mathrm{~mm}$ cylinder are initially analyzed and compared with the available experimental data. Both the Strouhal number, St, and the velocity profile in the wake of the cylinder are considered.

\subsubsection{Strouhal number, $S t$}

The action of alternate vortex shedding results to fluctuations of the lift forces due to pressure variations on the cylinder and fluctuations of the velocity as the vortices travels through the wake. From the simulation results, $S t$ can be determined from both these parameters. The velocity fluctuations are obtained by subtracting the average velocity from the instantaneous velocity at a point in the wake of cylinder. In this case, the velocity is obtained at a distance of two cylinder diameters downstream of the cylinder and at a height of $0.00925 \mathrm{~m}$ from the pipe bottom. Although both velocity components would provide similar fluctuation frequency, the vertical velocity component was considered because it had larger fluctuations. For the case of $u=0.650 \mathrm{~m} / \mathrm{s}$ with corresponding $R e_{D}=3250$, the fluctuating ferquencies, $f_{v}$, were found to be $27.1 \mathrm{~Hz}$ and $26.32 \mathrm{~Hz}$ for lift coefficient and the fluctuating velocities respectively giving $S t$ equal to 0.208 and 0.202. These St agree with the experimental data from Chinaud et al. [7] and are very close to the value of 0.20 for unbounded flows. 


\subsubsection{Velocity profile}

The simulated average velocity profiles for the flow past the $5 \mathrm{~mm}$ cylinder are further compared with the experimental data of Chinaud et al. [7] in Figure 1. The profiles are at a distance of two cylinder diameters ( 0 to $2 \mathrm{D}$ or $0 \mathrm{~mm}$ to $10 \mathrm{~mm}$ ) from the cylinder and at the bottom half of the pipe. Two cases with different Reynolds numbers based on the cylinder diameter, $R e_{D}$, are considered, namely $R e_{D}=1192$ and $R e_{D}=3250$. In Figure 1 the center of the cylinder is located below the pipe centre at $y=-0.00925 \mathrm{~m}$ with the pipe center at $y=0$. The velocity profiles in this case are averaged over 10 cycles of vortex shedding. Good agreement is observed between the simulated and the experimental data with average deviation of less than $6 \%$.

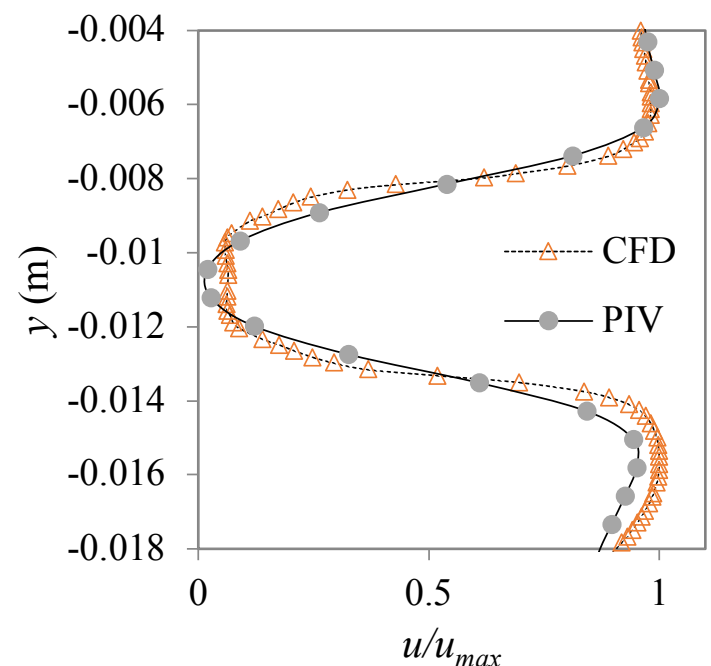

(a) $\operatorname{Re}_{D}=1192$

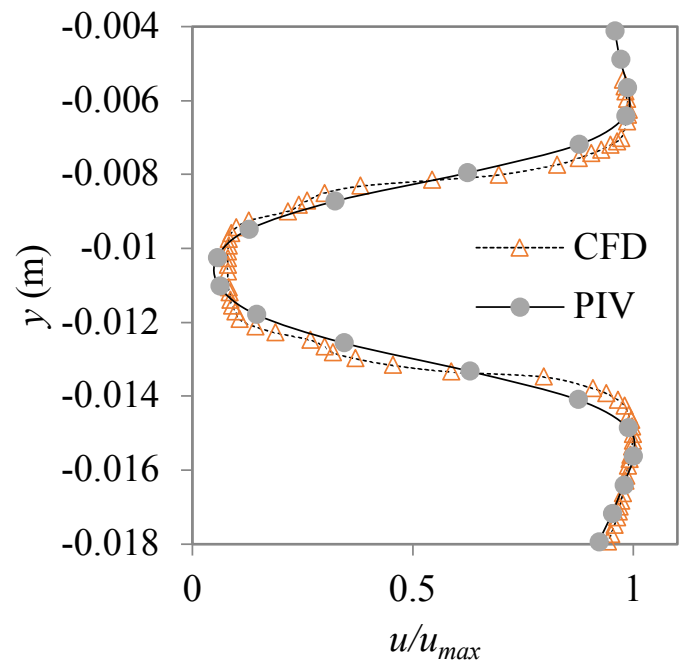

(b) $R e_{D}=3250$

Figure 1: Comparisons of velocity profiles behind the $5 \mathrm{~mm}$ cylinder in single phase water flow obtained with CFD and with PIV. PIV data from Chinaud et. al. [7].

The agreement between the simulations and the experimental data demonstrated that the simulations are able to reproduce the wake from the cylinder at least in the middle plane of the pipe. Grid sensitivity analysis showed that when $250 \mathrm{k}$ cells was used, the Strouhal numbers calculated were approximately $3.8 \%$ different from those found with a coarser grid $(150 \mathrm{k})$, while the computation time was doubled. Therefore, subsequent simulations were conducted with 150k cells; the details of the numerical setup are shown in Table 1. 
Table 1: Details of the numerical simulations

\begin{tabular}{cc}
\hline Numerical Setup & Type/Value \\
\hline Turbulent Model & $k-\varepsilon$ with enhanced wall \\
treatment \\
Pressure-Velocity & SIMPLE algorithm \\
Coupling & \\
Spatial Discretization & Second Order Upwind \\
Step Size & $0.0005-0.001 \mathrm{~s}$ \\
No. Of Steps & $2000-4000$ \\
Cell Counts & $150 \mathrm{k}$ \\
\hline
\end{tabular}

2.1.3 Investigation of flow past cylinder of various diameters

From the validated two-dimensional single-phase model developed for the $5 \mathrm{~mm}$ cylinder, further simulations were performed to investigate the vortex shedding frequencies of cylinders with different diameters. Five cylinder diameters were considered, namely 2, 7, 8, 9 and $10 \mathrm{~mm}$ and the summary of simulation results is presented in Table 2. 
Table 2: Simulation results for various cylinder diameters

\begin{tabular}{|c|c|c|c|c|c|}
\hline $\begin{array}{c}D \\
(\mathrm{~mm})\end{array}$ & $\begin{array}{l}\gamma \\
(-)\end{array}$ & $\begin{array}{c}u \\
(\mathrm{~m} / \mathrm{s})\end{array}$ & $\operatorname{Re}_{D}$ & $\begin{array}{c}f_{v} \\
(\mathrm{~Hz})\end{array}$ & $\begin{array}{l}S t \\
(-)\end{array}$ \\
\hline \multirow[t]{3}{*}{2} & 4.125 & 0.310 & 620 & 29.85 & 0.21 \\
\hline & & 0.620 & 1240 & 71.43 & 0.22 \\
\hline & & 1.085 & 2170 & 117.20 & 0.23 \\
\hline \multirow[t]{3}{*}{5} & 1.350 & 0.310 & 1550 & 10.00 & 0.21 \\
\hline & & 0.620 & 3100 & 26.26 & 0.20 \\
\hline & & 1.085 & 5425 & 46.29 & 0.23 \\
\hline \multirow[t]{3}{*}{7} & 0.821 & 0.310 & 2170 & 8.87 & 0.21 \\
\hline & & 0.620 & 4340 & 21.05 & 0.23 \\
\hline & & 1.085 & 7595 & 34.00 & 0.23 \\
\hline \multirow[t]{3}{*}{8} & 0.656 & 0.310 & 2480 & 8.00 & 0.21 \\
\hline & & 0.620 & 4960 & 19.31 & 0.23 \\
\hline & & 1.085 & 8680 & 34.00 & 0.28 \\
\hline \multirow[t]{3}{*}{9} & 0.528 & 0.310 & 2790 & 8.00 & 0.23 \\
\hline & & 0.620 & 5580 & 16.00 & 0.23 \\
\hline & & 1.085 & 9765 & 32.00 & 0.28 \\
\hline \multirow[t]{3}{*}{10} & 0.425 & 0.310 & 3100 & 8.00 & 0.26 \\
\hline & & 0.620 & 6200 & 18.58 & 0.27 \\
\hline & & 1.085 & 10850 & 40.00 & 0.28 \\
\hline
\end{tabular}

As can be seen from Table 2, the cylinder diameters considered varying from $2 \mathrm{~mm}$ to $10 \mathrm{~mm}$ are able to expand the range of vortex shedding frequencies, $f_{v}$, over two orders of magnitude from 1 to $100 \mathrm{~Hz}$. In the majority of cases, the Strouhal numbers are similar to unbounded flow. However, for the case of $u=1.085 \mathrm{~m} / \mathrm{s}$ for cylinder diameters of 8 and $9 \mathrm{~mm}$ and for all velocities tested for the $10 \mathrm{~mm}$ cylinder, an increase in St was observed. The increase in St implies that wall effects become significant and affect vortex shedding as the gap ratio, $\gamma$, is reduced with increasing $D$. As the simulation is two-dimensional, the wall effects could more profound in the experimental investigations in the pipe. Therefore, for the experimental studies, the 2 $\mathrm{mm}$ and $8 \mathrm{~mm}$ cylinders are selected to investigate the effects of varying vortex properties on the interfacial waves and on the transition from stratified to non-stratified flow patterns. A schematic of the size and position of the cylinders in the pipe is shown 
in Figure 2. The center of each cylinder is located $9.25 \mathrm{~mm}$ from the bottom pipe. The corresponding gap ratios, $\gamma$ for the cylinder diameters are presented in Table 3.
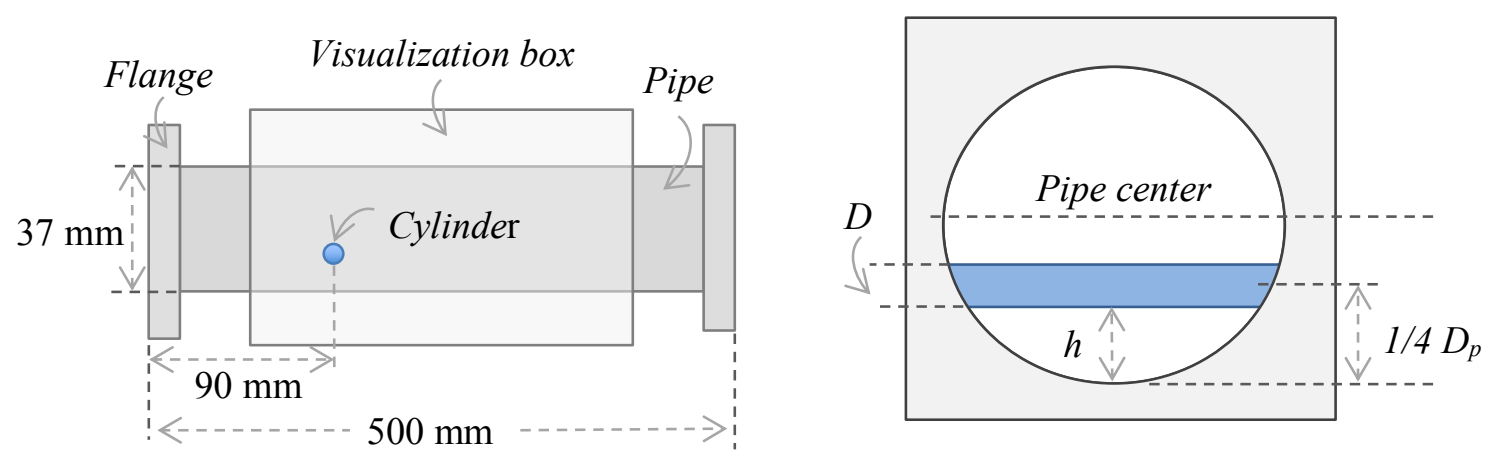

Figure 2: Schematic of the pipe section containing the bluff body (a) Side view and (b) cross section view

Table 3: Diameters of cylinders and corresponding gap ratios from the bottom pipe wall

\begin{tabular}{ccc}
\hline$D(\mathrm{~mm})$ & $h(\mathrm{~mm})$ & $\gamma(-)$ \\
\hline 2 & 8.25 & 4.12 \\
$5^{*}$ & 6.75 & 1.35 \\
8 & 5.25 & 0.66 \\
\hline & * Park et al., [6]
\end{tabular}

\subsection{Experimental setup}

The experiments were conducted in the water-oil flow facility presented in Figure 3. The test section is made of $37 \mathrm{~mm}$ ID and $8 \mathrm{~m}$ long clear acrylic pipes which allows the flow to be observed and recorded. The two fluids are introduced into the test section via a symmetrical Y-shaped junction (20 $0^{\circ}$ angle between branches) designed to minimize mixing. The transverse cylinder is located about $0.20 \mathrm{~m}$ downstream of the Y-junction and the pipe section that contains the cylinder is enclosed in an acrylic visualization box filled with glycerin to reduce optical distortions and enhance image quality. After the test section, the two phases are separated in a gravity settler equipped with a KnitMesh ${ }^{\mathrm{TM}}$ coalescer and they flow by gravity in their respective storage tanks. The two fluids are driven separately from their tanks via centrifugal pumps to the inlet of the test section. The flow rates are regulated through an automated flowrate controller (Allen Bradley-Panel View C400) connecting the flowmeters to the pumps 
(Flowserve). The test fluids are water and Exxsol D140 oil (density, $\rho=830 \mathrm{~kg} / \mathrm{m}^{3}$ and viscosity, $\mu=0.0055 \mathrm{~kg} / \mathrm{ms}$ ). Since the refractive index of the two liquids is not matched, PIV measurements in the water phase are only carried out for the cases where the interface is not very disturbed. Low flowrates of the two phases were chosen which resulted in linear waves downstream of the cylinder. At these flowrates the liquid-liquid interface approaching the cylinder was also almost flat. The two-phase flows in this work are described by their mixture velocity, $u_{\text {mix }}$ and input oil to water flowrate ratio, $r$, as shown in Eq. 4 and Eq. 5 where $Q_{o}$ and $Q_{w}$ are the volumetric flowrates of the oil and water phases respectively, while $R$ is the radius of the pipe.

$$
u_{m i x}=Q_{o}+Q_{w} / \pi R^{2}
$$

$$
r=Q_{o} / Q_{w}
$$

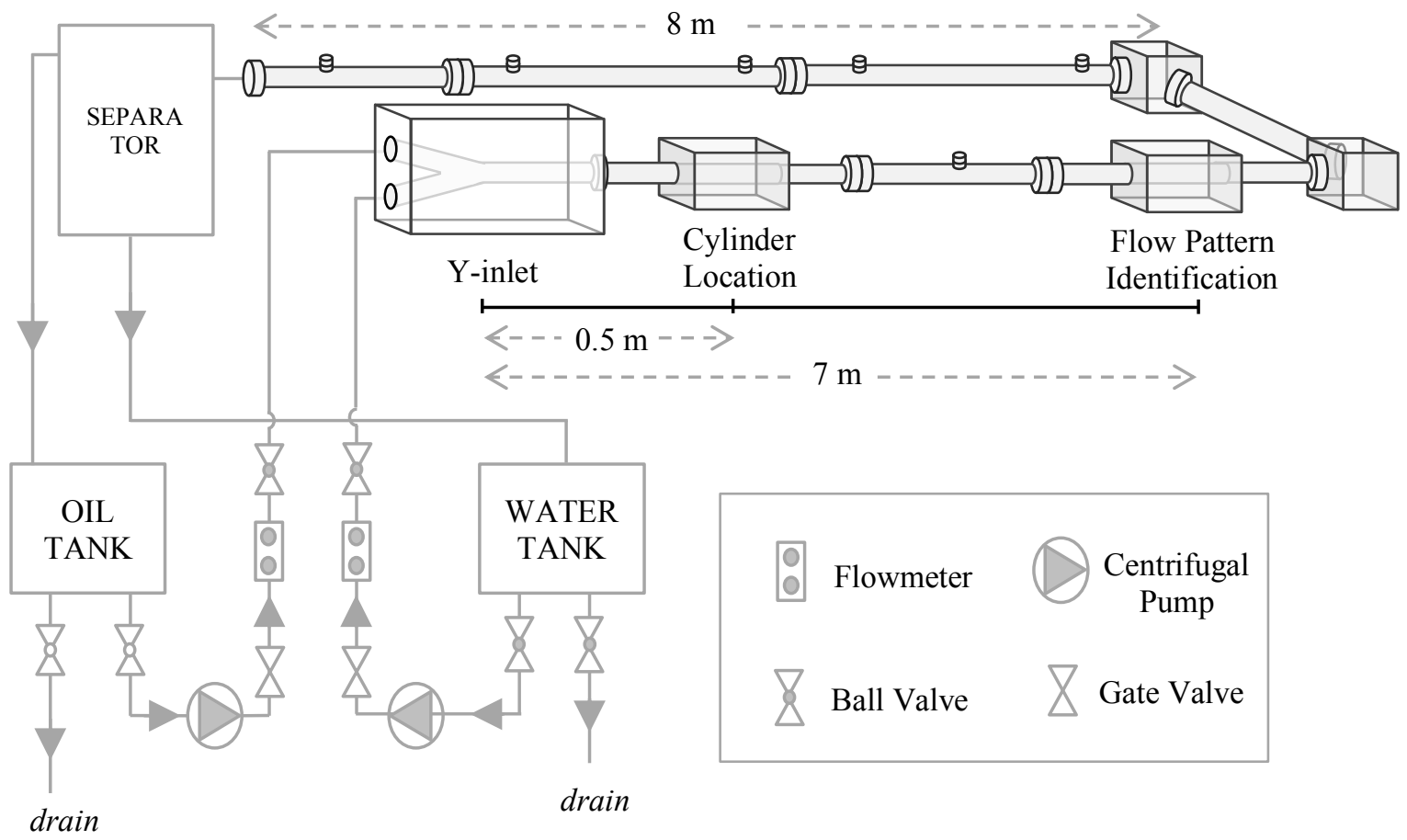

Figure 3: Schematic of the two-phase oil-water flow facility

2.3 Measurement techniques

\subsubsection{High-speed imaging}

The properties of interfacial waves of the two phase flow in the test section were observed and recorded via high-speed imaging. A Photron SA-1 camera with Sigma 
$105 \mathrm{~mm} \mathrm{f} / 2.8$ macro lens was used at a frame rate ranging from 2000 to $5000 \mathrm{fps}$ depending on the flowrates, with full resolution of 1024 x 1024 pixels. The test section was illuminated with a backlight as shown in Figure 4(a). Visualizations were conducted at two locations along the pipe test section, in the vicinity of the bluff body ( $0.2 \mathrm{~m}$ downstream of the cylinder) and at $7 \mathrm{~m}$ downstream of the $Y$-inlet to record the different flow patterns.

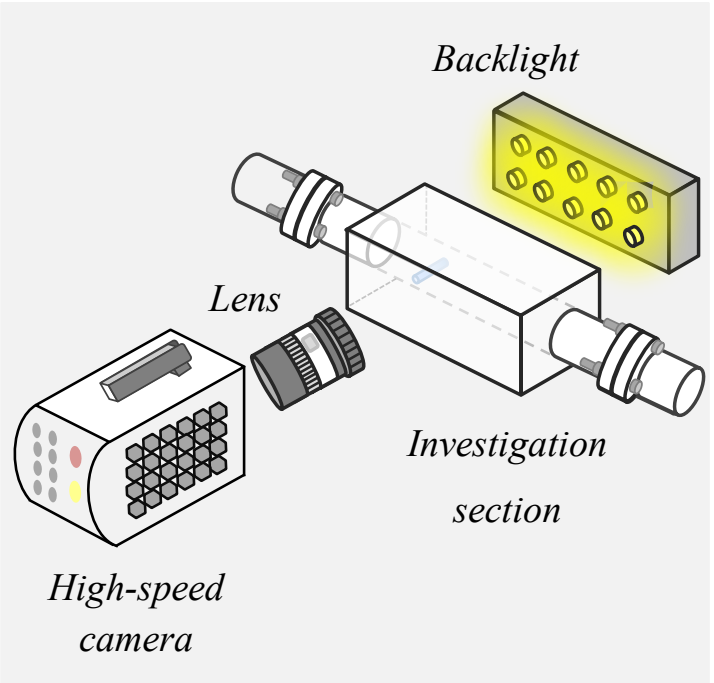

(a)

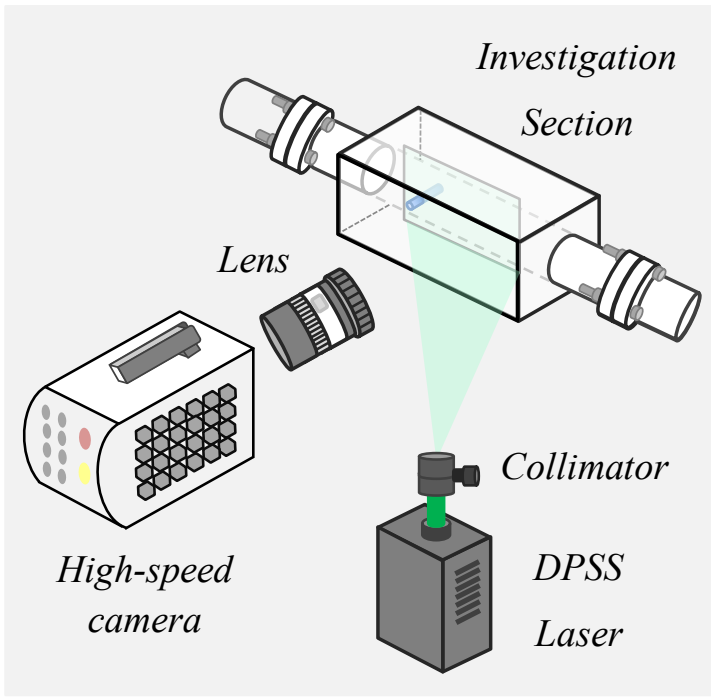

(b)

Figure 4: Equipment set up (a) High-speed visualization and (b) PIV

\subsubsection{Particle image velocimetry (PIV)}

PIV measurements were conducted to determine the velocity profiles in the vicinity of the bluff body for both single and two-phase flows. To improve the resolution in the wake of the bluff body, the magnification of the camera was increased at a distance of $0.1 \mathrm{~m}$ behind the cylinder. The water phase was seeded with silver coated hollow glass particles with $10 \mu \mathrm{m}$ diameter (TSI instruments). A DPSS Continuous Laser (Laserglow Technologies, emission wavelength at $532 \mathrm{mn}$ and power at $3000 \mathrm{~mW}$ ) was used to illuminate the flow from underneath the pipe. With a classical expanded laser beam optical system, a light sheet was generated with estimated thickness of $1 \mathrm{~mm}$, as shown in Figure 4 (b). The optical system was arranged so that the light sheet is located vertically in the middle of the pipe section along the pipe axis. Images were recorded with the high-speed camera in full field of $1024 \times 1024$ pixels, located perpendicularly to the laser sheet. Depending on the flow rate, the sampling frequencies which are 
controlled by the camera frame rate were varied between 3000 to $5000 \mathrm{fps}$. This corresponds to time differences $\delta$ t from 0.20 to $0.33 \mathrm{~ms}$ between the image pairs used to calculate the velocity vectors. For each set of conditions, 6000 to 10000 images were acquired corresponding to a flow time of $2 \mathrm{~s}$. The captured images were treated with JPIV software, where an adaptive correlation tracking was used to obtain the two dimensional velocity fields using a $16 \times 16$ pixel correlation box with $50 \%$ spatial overlap. The pixel size was $100 \mu \mathrm{m}$ which gives a spatial resolution for the velocity vector of $0.8 \mathrm{~mm}$ (half the correlation box).

\subsection{Results and discussion}

In this section, the results of the PIV measurements in single phase water flow are first discussed followed by the findings in the two-phase oil-water flows. In single phase flow, the vortex shedding characteristics in the wake of the cylinders are analyzed. For the two-phase flows, the flow patterns established at $7 \mathrm{~m}$ downstream the pipe are discussed in relation to the cylinder size. The vortex shedding characteristics and the resulting oil-water interfacial waves in the vicinity of the bluff body are then analyzed from the high-speed visualization and the PIV measurements.

\subsection{Single-phase flows}

The vortex shedding characteristics in single-phase water flows were investigated with PIV for the two cylinder diameters $(2 \mathrm{~mm}$ and $8 \mathrm{~mm}$ ) chosen based on the CFD simulations.

\subsubsection{Vortex shedding frequency}

To compute the vortex shedding frequencies from the cylinders, FFT analysis of the vertical velocity, $v$, was performed at a distance of five cylinder diameters behind the cylinder and at the same vertical location as the cylinder centre. This location is chosen because it is at an area where vortices pass through as they travel downstream the cylinder and therefore has high radial velocities. Figure 5 shows an example of $v$, taken in the wake of the $2 \mathrm{~mm}$ and $8 \mathrm{~mm}$ cylinders. The $f_{v}$ is then used to calculate the corresponding Strouhal number and the results are presented in Table 4. 


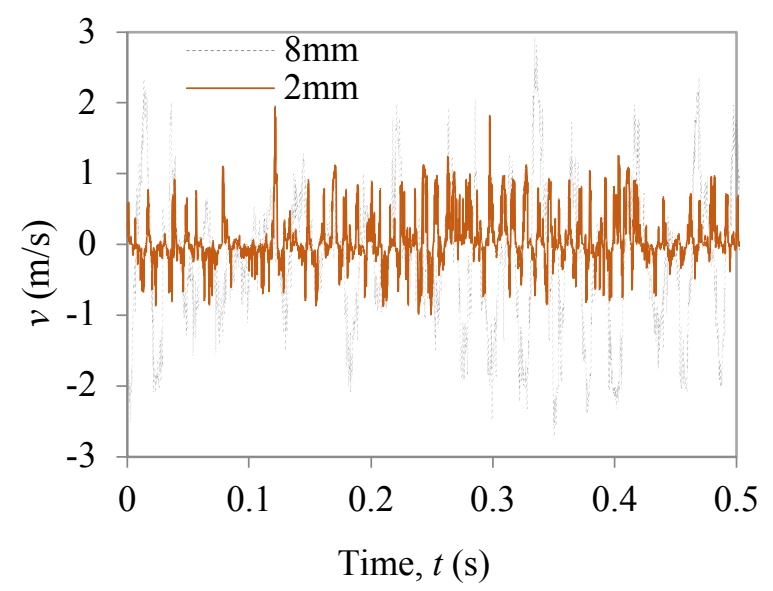

Figure 5: Vertical velocity $\mathrm{r}$ at a location in the wake of the 2 and $8 \mathrm{~mm}$ cylinders for single phase water flow at $u=1.085 \mathrm{~m} / \mathrm{s}$

Table 4: Vortex shedding frequencies from the cylinders and St for single phase flows

\begin{tabular}{|c|c|c|c|c|c|c|c|c|}
\hline \multirow{2}{*}{$\begin{array}{c}Q \\
(\mathrm{~L} / \mathrm{min})\end{array}$} & \multirow{2}{*}{$\begin{array}{c}u \\
(\mathrm{~m} / \mathrm{s})\end{array}$} & \multirow{2}{*}{$R e_{\text {pipe }}$} & \multicolumn{3}{|c|}{$D=2 \mathrm{~mm}, \gamma=1.35$} & \multicolumn{3}{|c|}{$D=8 \mathrm{~mm}, \gamma=0.66$} \\
\hline & & & $R e_{D}$ & $f_{v}(\mathrm{~Hz})$ & $S t$ & $R e_{D}$ & $f_{v}(\mathrm{~Hz})$ & $S t$ \\
\hline 10 & 0.155 & 5735 & 310 & 14.72 & 0.19 & 1240 & 5.43 & 0.28 \\
\hline 20 & 0.310 & 11470 & 620 & 29.30 & 0.19 & 2480 & 10.94 & 0.28 \\
\hline 30 & 0.465 & 17205 & 930 & 45.35 & 0.20 & 3720 & 16.42 & 0.28 \\
\hline 40 & 0.620 & 22940 & 1240 & 60.49 & 0.20 & 4960 & 21.35 & 0.27 \\
\hline 50 & 0.774 & 28638 & 1548 & 73.90 & 0.19 & 6192 & 26.22 & 0.27 \\
\hline 60 & 0.929 & 34373 & 1858 & 88.67 & 0.19 & 7432 & 31.12 & 0.27 \\
\hline 70 & 1.085 & 40108 & 2168 & 108.71 & 0.20 & 8672 & 37.86 & 0.28 \\
\hline
\end{tabular}

From Table 4, the range of vortex shedding frequencies achieved with the investigated cylinder diameters is between 5 and $108 \mathrm{~Hz}$. The results agree well with the numerical studies of two-dimensional flows, indicating that at least in the middle of the pipe the flow was not affected by the three dimensional pipe geometry. For the flow velocities studied, the $2 \mathrm{~mm}$ cylinder generates vortices with higher frequency (14.72 to 108.71 $\mathrm{Hz}$ ) compared to the $8 \mathrm{~mm}$ cylinder $(5.43$ to $37.86 \mathrm{~Hz})$. At these flow conditions, the vortex shedding frequencies are proportional to the incoming water velocity. The average Strouhal number, $S t=0.20$, for the $2 \mathrm{~mm}$ cylinder matches the literature value 
for unbounded flows, which suggests that the system resembles unbounded flow at least in the middle of the pipe.

An increase in $S t$ was observed for the $8 \mathrm{~mm}$ cylinder, with an average value of 0.28 . This is related to the reduced gap ratio of $\gamma=0.66$ for this cylinder size and to the effect of the wall on the frequency of the vortices. This suggests that interactions occur between the wall boundary layer and the vortices behind the cylinder as observed by Patil and Tiwari [10] and Rehimi et al. [14]. An increase in the frequency of the vortices downstream of a bluff body in the vicinity of a wall compared to unconfined flows was also reported by Zovatto and Pedrizetti [13]. Renfer et al. [34] reported that the frequencies of vortex produced from micropin arrays increased with decreasing gap ratio. To elucidate the wall effects on vortex shedding, the vorticity structures obtained from the PIV measurements are analyzed in the next section.

\subsubsection{Vortex shedding characteristics: Vorticity}

The spanwise vorticity, $\omega_{z}$, is computed to visualize the vortical structures generated behind the cylinder and is determined from the following equation:

$$
\omega_{z}=\frac{\partial v}{\partial x}-\frac{\partial u}{\partial y}
$$

In Eq. 6, $u$ and $v$ are the horizontal and vertical velocity components. The instantaneous vorticity fields in the wakes of the cylinders are shown in Figure 6(a) and (b) for a flowrate of $30 \mathrm{~L} / \mathrm{min}$. The $y$-axis represents the pipe diameter with the bottom of the pipe positioned at $y=0$. The scale has been increased in the y-direction to better show the vortices forming and for this reason the cylinders appear elliptical. Vortices are shed alternatingly behind each cylinder and are convected downstream, with the positive vorticity localized above the cylinder and the negative one below. This is in agreement with literature $[7,13]$ as the Reynolds numbers investigated in this work are above the critical value $\left(R e_{D}=40\right)$ for vortex shedding to occur. The vorticity magnitude in the wake declines with increasing distance away from the cylinder. It can also be seen from Figure 6 that larger vortices are shed from the $8 \mathrm{~mm}$ cylinder compared to the $2 \mathrm{~mm}$ one. In addition, the vortices behind the $8 \mathrm{~mm}$ cylinder are sustained further downstream the flow. 

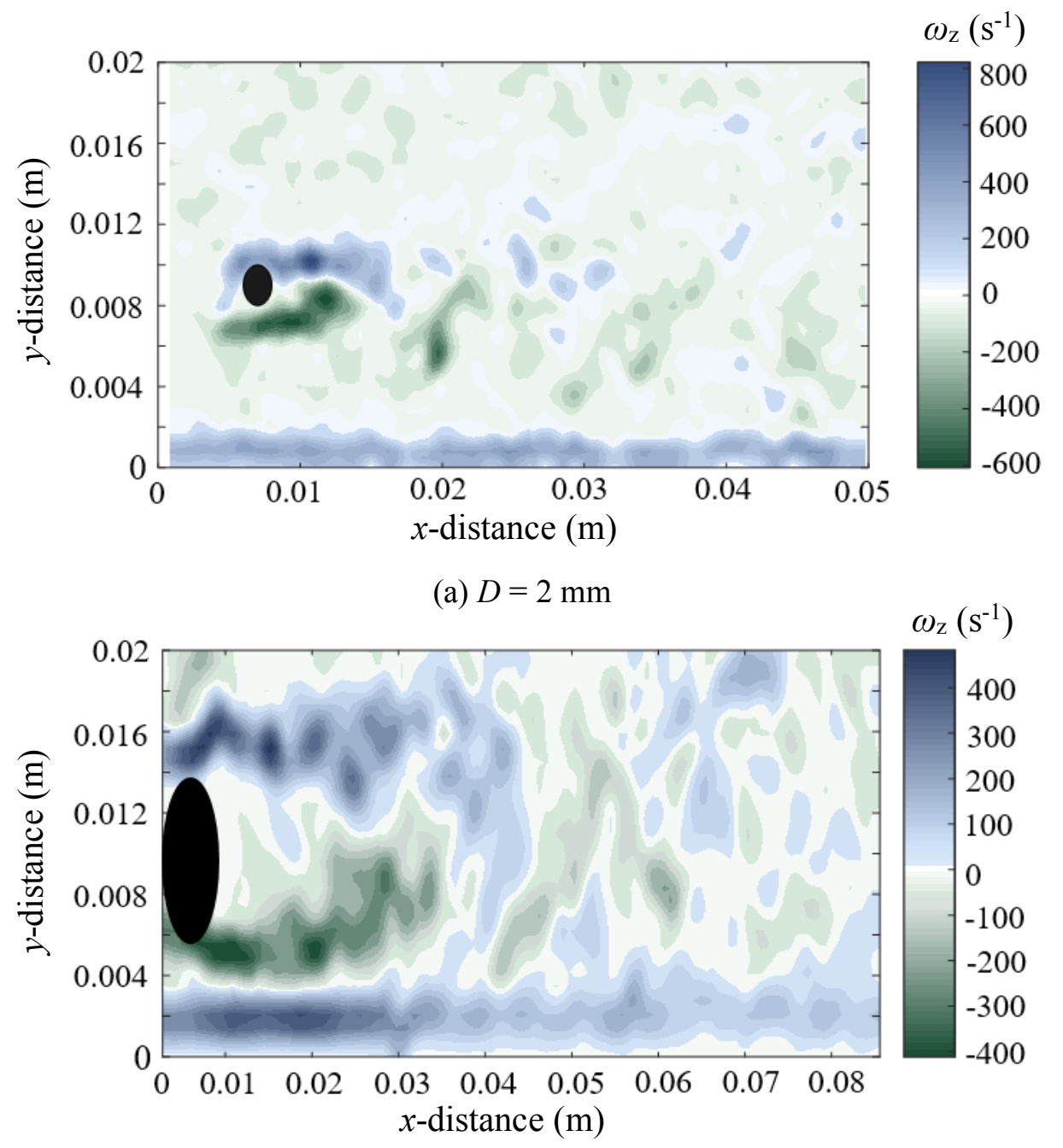

(b) $D=8 \mathrm{~mm}$

Figure 6: Vorticity field in the wake of cylinders with different diameters for single phase water flow at $\mathrm{Q}_{\mathrm{w}}=30 \mathrm{~L} / \mathrm{min}, u=0.465 \mathrm{~m} / \mathrm{s}$.

The time averaged vorticities computed for one period of vortex shedding at different velocities are presented in Figures 7 and 8 for the $2 \mathrm{~mm}$ and $8 \mathrm{~mm}$ cylinders respectively. As expected, for both cylinders, the main vorticity structures formed on the top and at the bottom of the cylinder. In Figure 7, for the case of $D=2 \mathrm{~mm}$ where the gap ratio is $\gamma=4.12$, the vorticity structures are symmetric and resemble those of the unbounded flow case. When the incoming velocity is increased from $0.465 \mathrm{~m} / \mathrm{s}$ to $0.929 \mathrm{~m} / \mathrm{s}$, the magnitude of the vorticity increases, as seen in Figure 7(b), but the vorticity field structures remain similar. 


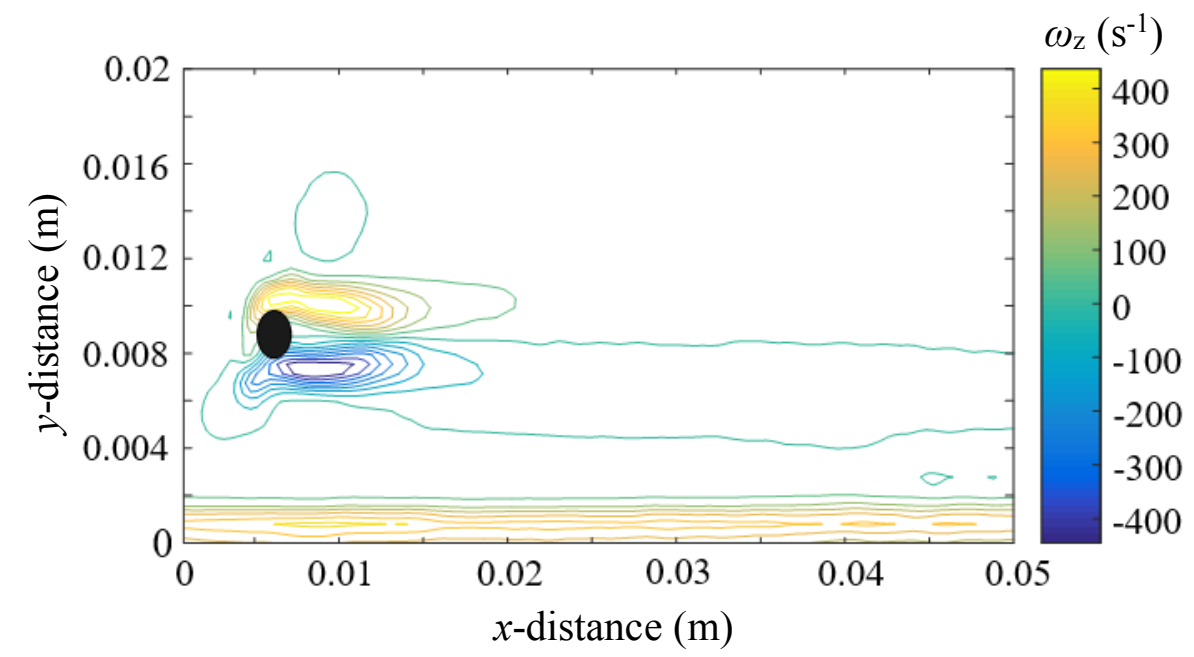

(a) $u=0.465 \mathrm{~m} / \mathrm{s}$

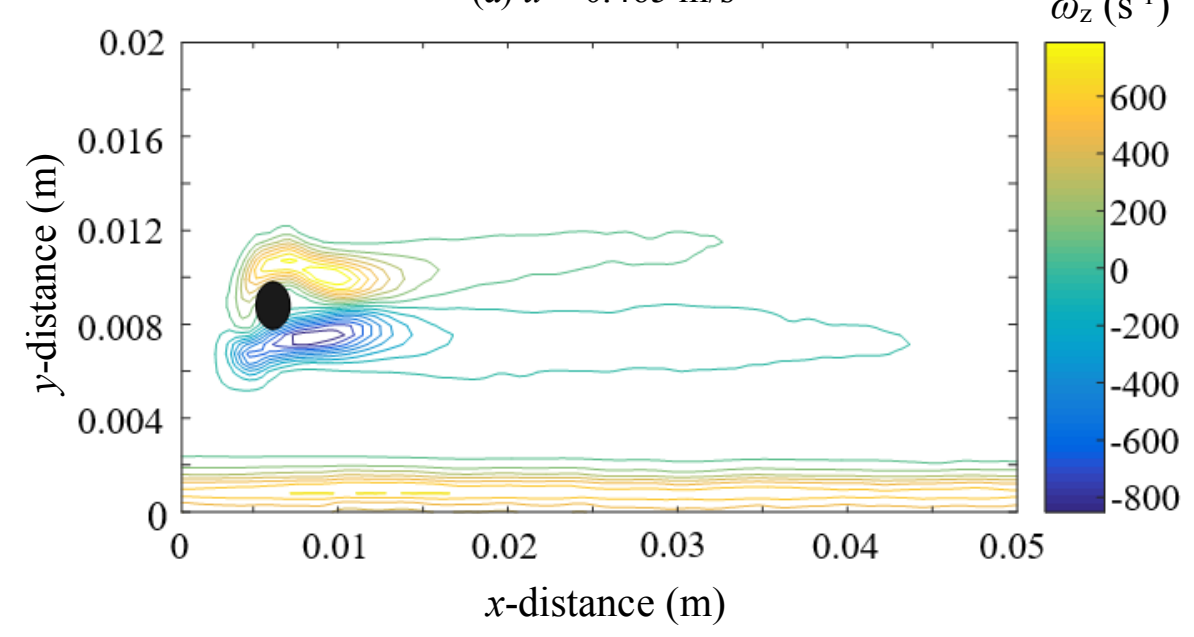

(b) $u=0.929 \mathrm{~m} / \mathrm{s}$

Figure 7: Time averaged vorticity in the wake of the $2 \mathrm{~mm}$ cylinder for single phase water flow at different velocities

For the case of $D=8 \mathrm{~mm}$ in Figure 8 , the distance between the cylinder and the bottom pipe wall is smaller with $\gamma=0.66$. The vorticity structures are larger and spread further behind the cylinder compared to the $2 \mathrm{~mm}$ cylinder case. The wake structure is not symmetric and the vortices at the upper part of the cylinder are longer than those at the bottom part. This asymmetry is attributed to the presence of the wall, which generates vorticity at the wall boundary layer that interacts with the vorticity at the lower part of the cylinder. The effects of the wall on the wake structure are more pronounced at the higher velocity of $0.93 \mathrm{~m} / \mathrm{s}$, as shown in Figure $8(\mathrm{~b})$. The asymmetric wake structure which occurs at low gap ratios is in agreement with the numerical findings of Zovatto \& Pedrizzetti [13]. 

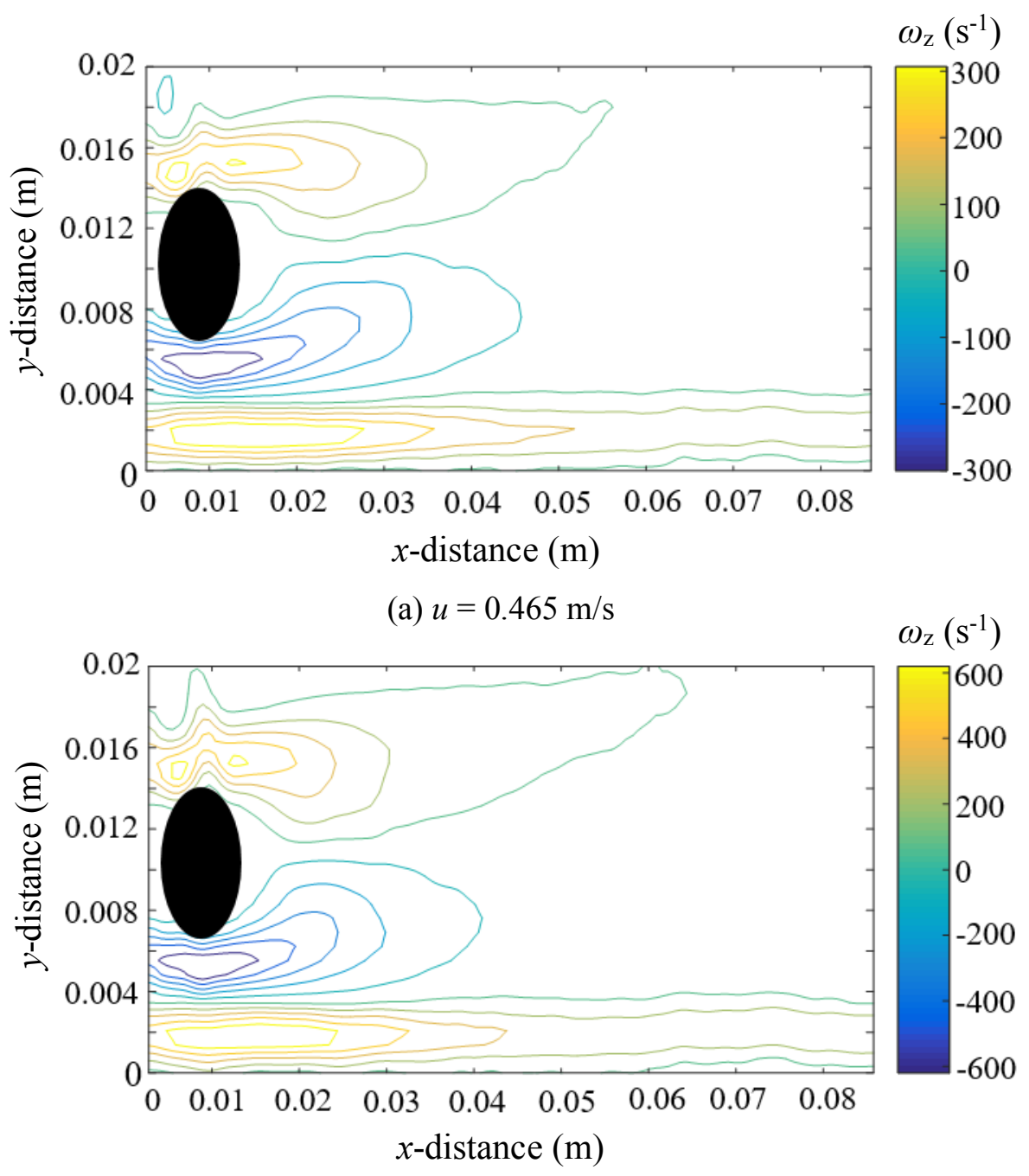

(b) $u=0.929 \mathrm{~m} / \mathrm{s}$

Figure 8: Time averaged vorticity in the wake of the $8 \mathrm{~mm}$ cylinder for single phase water flow at different velocities

\subsection{Two-phase flows}

In two-phase flow, the flow patterns established when the bluff bodies are present in the pipe were identified at $7 \mathrm{~m}$ downstream of the inlet via high-speed imaging. In addition, recordings were carried out in the vicinity of the bluff body $(0.2 \mathrm{~m}$ behind cylinder) to characterize the generated interfacial waves. In this region, velocity fields were also acquired from PIV measurements in the water phase to determine the properties of the vortices generated by the cylinders and their interactions with the oilwater interface. 


\subsubsection{Flow patterns in two-phase flows}

Three types of flow patterns were identified in this work, namely stratified wavy (SW), dual continuous with oil drops in water (DC \& O/W) and dual continuous with drops of both phases dispersed into the opposite one (DC), with representative images shown in Figure 9. These patterns are commonly encountered during the flow of two immiscible liquids with small viscosity difference. The corresponding flow pattern maps for the $2 \mathrm{~mm}$ and $8 \mathrm{~mm}$ cylinders are presented in Figure 10. The transition boundaries from stratified to dual continuous flow are also shown as lines for the systems without a cylinder and with a $5 \mathrm{~mm}$ cylinder [6], for reference.

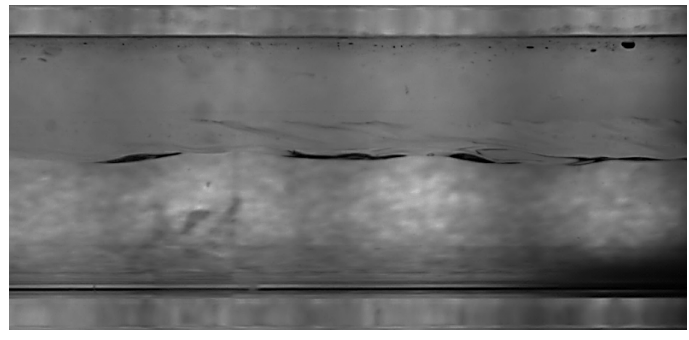

Stratified Wavy, $\boldsymbol{S W}$

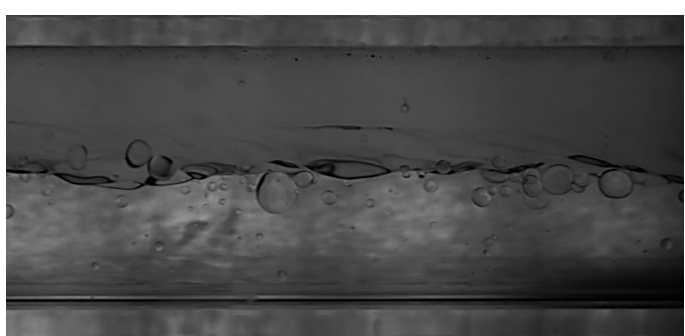

Dual continuous with oil and water drops, $\mathrm{DC}(\mathrm{O} / \mathrm{W}$ and $\mathrm{W} / \mathrm{O})$

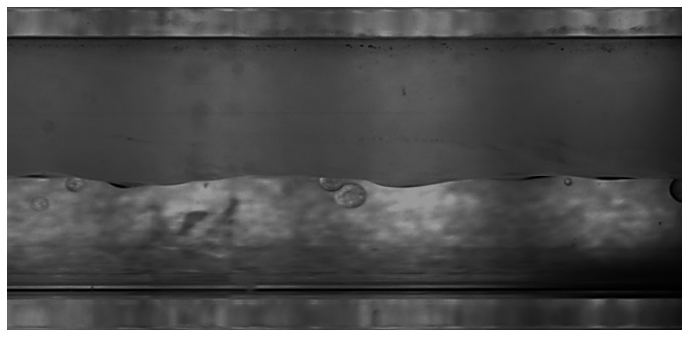

Dual continuous with oil drops, $\boldsymbol{D C}(\boldsymbol{O} / \mathbf{W})$

Figure 9: Oil-water flow patterns at $7 \mathrm{~m}$ downstream of the pipe inlet 


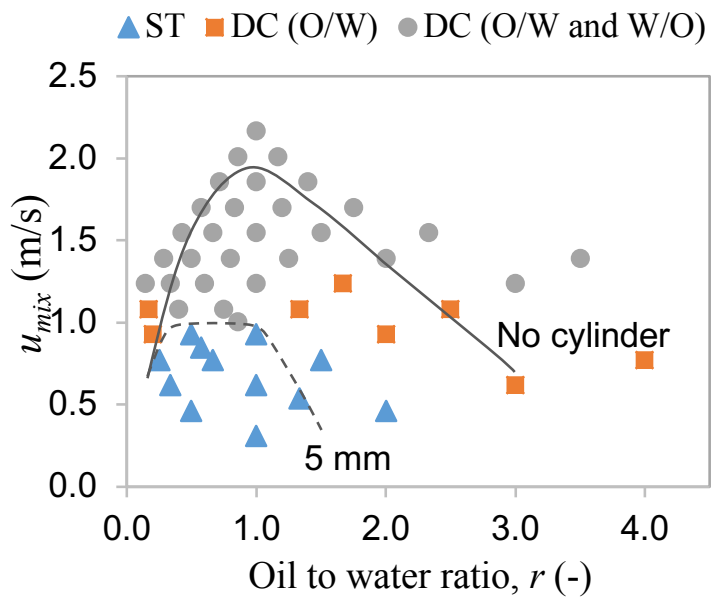

(a) $D=2 \mathrm{~mm}$

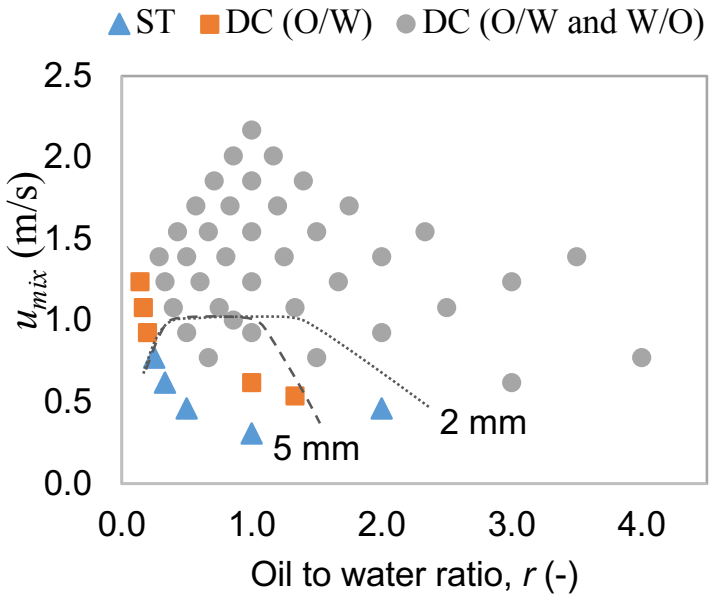

(b) $D=8 \mathrm{~mm}$

Figure 10: Flow pattern map oil-water flows at $7 \mathrm{~m}$ downstream of the pipe inlet for cylinder sizes (a) $D=2 \mathrm{~mm}$ and (b) $D=8 \mathrm{~mm}$. Transition lines for a system without a cylinder (straight), $D=5 \mathrm{~mm}$ (dashed) and $\mathrm{D}=2 \mathrm{~mm}$ (dotted) are also shown.

In the flow pattern map in Figure 10(a), the transition boundary from stratified to dual continuous flow is located at lower mixture velocities for the systems with the transverse cylinders present, compared to the system without a cylinder. It can also be observed that for the system without cylinder, the transition occurs at the highest mixture velocity for oil to water flow rate ratio $r=1.0$. This is in agreement with the transition mechanism from separated to dispersed flows which involves the development of interfacial waves and the detachment of drops from the waves when their amplitudes reach a critical value [35]. In the absence of the cylinder, waves develop when there is velocity difference between the two phases based on the KelvinHelmholtz instability. At $r=1.0$, waves develop only at very high mixture velocities. The presence of a transverse cylinder in this work actuates and facilitates the flow pattern transition by generating 'artificial' interfacial waves, shifting the boundaries towards lower mixture velocities. In addition, the transition at $r=1.0$. where the flowrates of the two phases are equal does not occur at the highest mixture velocity anymore since the interfacial waves are generated by the cylinder and not by the difference in velocities between the two phases.

The transition boundary from stratified to dual continuous flow is shifted towards lower mixture velocities as the cylinder diameter is increased (Figure 10(b)). It should be noted that at the same flow conditions, the cylinder diameter also determines the 
submergence depth. This suggests that the vortical structures generated by the different cylinder diameters, as discussed previously for the single phase flow, affect the generation of interfacial waves and lead to variations of the transition boundaries.

At low oil to water ratio, $(r<0.5)$, for the systems with the $2 \mathrm{~mm}$ and $5 \mathrm{~mm}$ diameter cylinders in Figure 10(a), the transition occurs at mixture velocities from $0.75 \mathrm{~m} / \mathrm{s}$ to 1 $\mathrm{m} / \mathrm{s}$, similar to the system without a cylinder. At these conditions the average interface height approaching the cylinder is located in the upper half of the pipe, above 21.50 $\mathrm{mm}$ from the pipe bottom. This corresponds to submergence depths above $11.26 \mathrm{~mm}$ and $9.76 \mathrm{~mm}$ for cylinder diameters $2 \mathrm{~mm}$ and $5 \mathrm{~mm}$ respectively. The distance between the interface and the von Karman vortices in the wake of cylinder is large for any significant interaction to occur. Different effects of the $2 \mathrm{~mm}$ and $5 \mathrm{~mm}$ cylinders on the transition are observed for oil-to-water flowrate ratios above 0.5 , where the interface height and the submergence depth of the cylinders are reduced. At ratio equal to 0.5 both systems undergo transition at $u_{\text {mix }}=1 \mathrm{~m} / \mathrm{s}$. As the ratio is further increased to 1.5, the transition boundary for the $5 \mathrm{~mm}$ cylinder system lowers from $1 \mathrm{~m} / \mathrm{s}$ to $0.5 \mathrm{~m} / \mathrm{s}$ mixture velocity, while for the $2 \mathrm{~mm}$ cylinder system it remains at $1 \mathrm{~m} / \mathrm{s}$, and only decreases at higher flowrate ratios.

For the $8 \mathrm{~mm}$ cylinder system, transition from SW to DC flow occurred at very low mixture velocities, apart from the lowest oil-to-water flowrate ratio where the submergence depth was $8.42 \mathrm{~mm}$ and the vortices shed would not have affected the interface. For ratios up to $r=2.0$, stratified flow was only seen at the lowest mixture velocities tested. To further understand the effects of bluff body size on the flow pattern actuation, the velocity fields and the structure of the vortices shed by the cylinders were studied with PIV.

\subsubsection{Investigations of two-phase flows in the vicinity of the cylinder}

In this section, the characteristics of the interfacial waves and of the vortices in the vicinity of the bluff body are discussed. Figure 11 shows the investigated region behind the bluff body with the measuring locations used in the analysis of the high speed images. For the velocity and vorticity fields obtained with PIV, the cases discussed are limited to low velocities $\left(u_{\mathrm{mix}}<0.465 \mathrm{~m} / \mathrm{s}\right)$. This is because the difference in refractive index between the two liquids leads to mutiple light reflections on the interface which 
is wavy at high velocities. As a result, the PIV tracer particles near the interface cannot be easily detected.

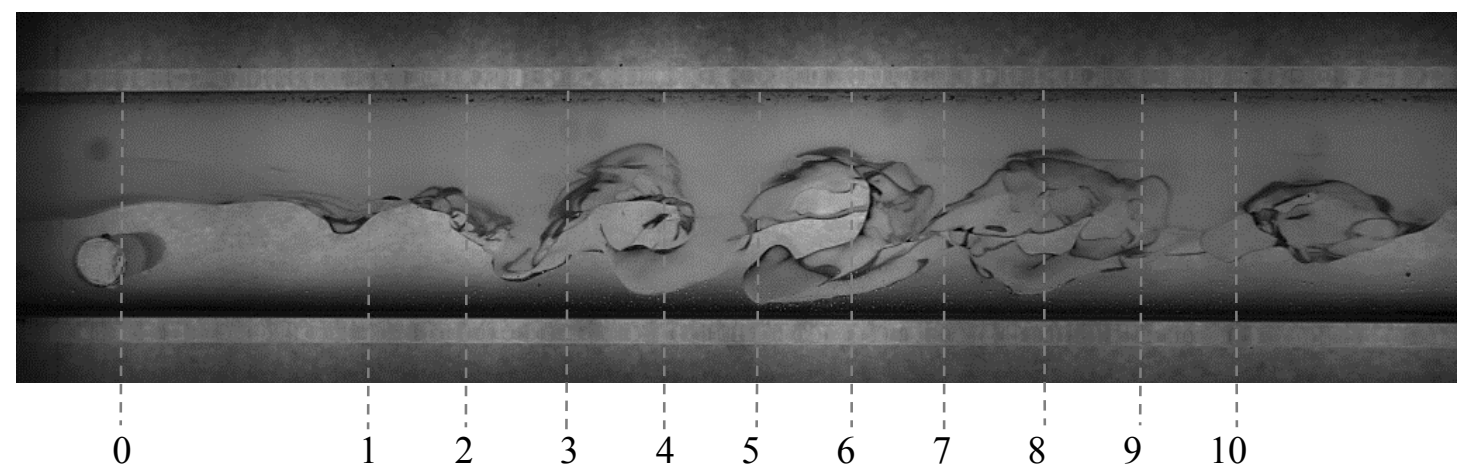

Figure 11: Measuring locations in the pipe downstream of the $8 \mathrm{~mm}$ cylinder.

Distance between locations 0 to 1 is $40 \mathrm{~mm}$ and between subsequent is $16 \mathrm{~mm}$.

\subsubsection{Vorticity in two-phase flows}

The instantaneous vorticity fields in the water phase are presented in Figures 12 and 13 for cylinder diameter of $2 \mathrm{~mm}$ and $8 \mathrm{~mm}$ respectively; the oil-water interface is also depicted in the figures as red line. Comparing the interface shapes it can be seen that the waves are more developed and have larger amplitude in the case of the large cylinder (Figure 13(a)) compared to the small cylinder (Figure 12) at the same water phase flow rates. The interface in the case of the $2 \mathrm{~mm}$ cylinder is almost flat with small fluctuations. This result is attributed to the combination of two factors, namely the cylinder submergence depth below the interface and the size of vortices generated. At constant oil to water ratio, the submergence depth is larger in the case of the small cylinder. As a result the interface is not very close to the the vortices behind the cylinder and is not significantly influenced by them; in addition the vortices generated in this case are smaller than with the $8 \mathrm{~mm}$ cylinder. 


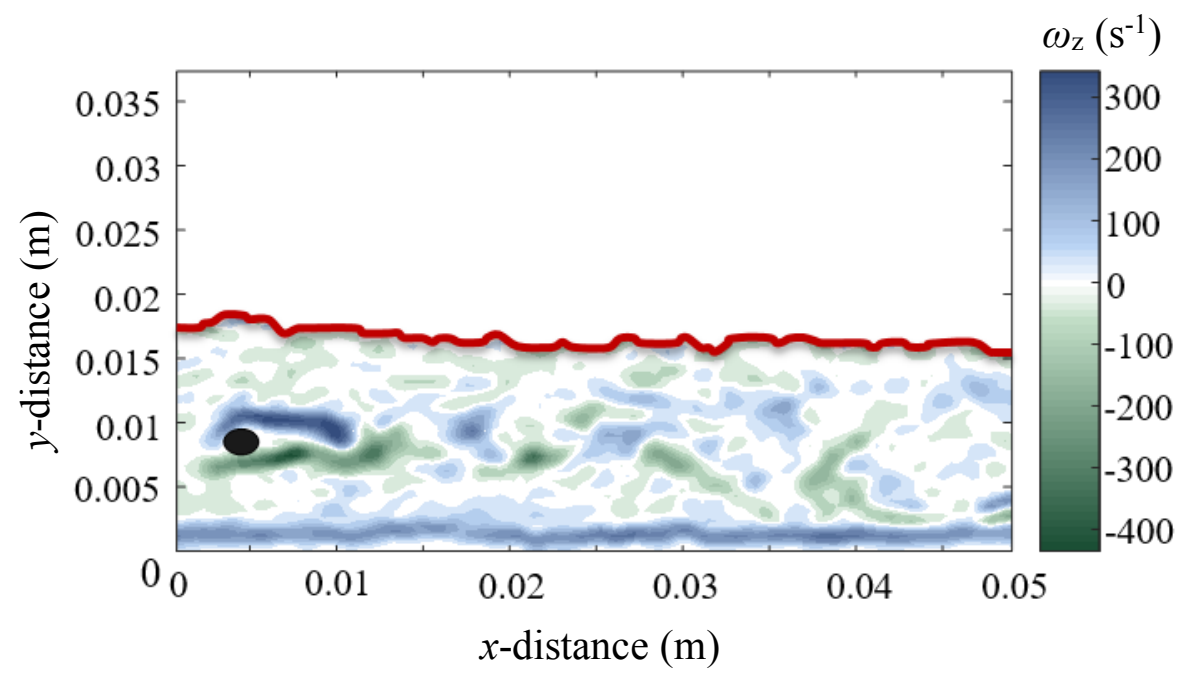

Figure 12: Vorticity field in the water phase of oil-water flows downstream of the $2 \mathrm{~mm}$ cylinder at $Q_{\mathrm{o}}=10 \mathrm{~L} / \mathrm{min}$ and $Q_{\mathrm{w}}=10 \mathrm{~L} / \mathrm{min}$. The interface is also shown as red line.

In the case of $D=8 \mathrm{~mm}$ cylinder (Figure 13(a)), the larger vortices lead to waves with larger amplitude. It can also be seen that the wave crests coincide with the locations of the positive vorticity regions shed from the upper side of the cylinder. The vortices on top of the cylinder are attached to the interface as soon as they are generated, actuating the formation of waves which then travel downstream as the vortices are convected with the flow. This supports the hypothesis that waves are generated at the frequency of vortex shedding. The results are in agreement with the work of Chinaud et. al. [7] which showed that vortices generated behind a cylinder in an oil-water flow are attached to interface. 


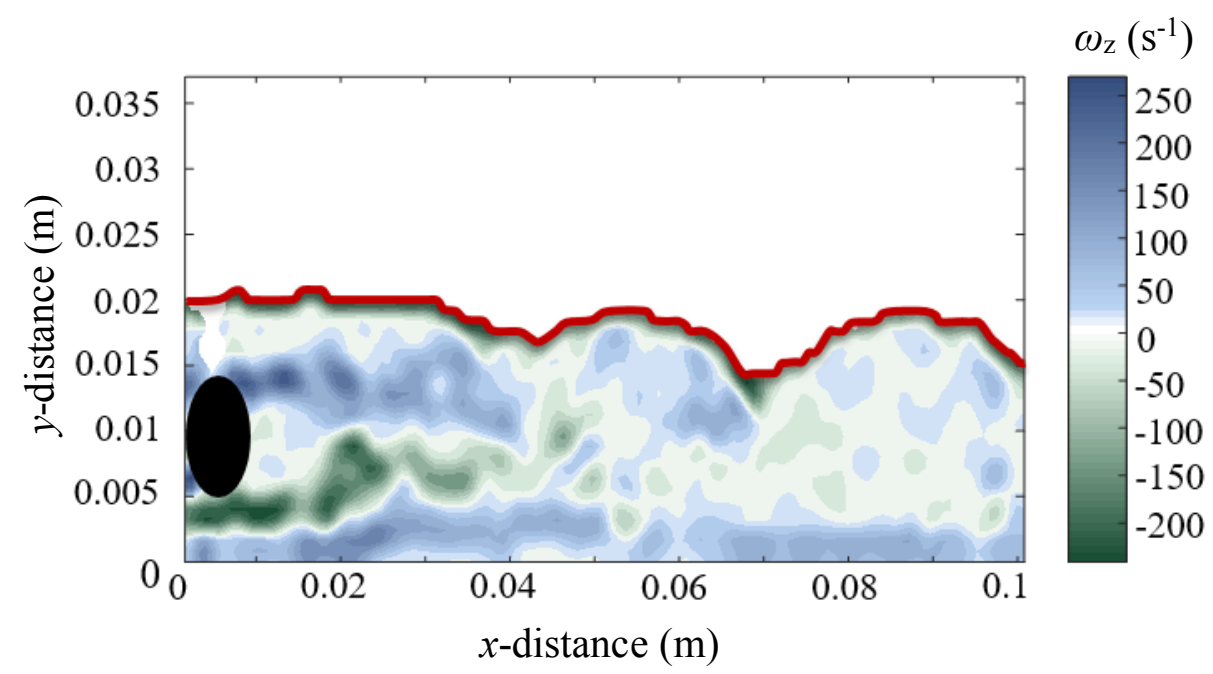

(a) $F r=1.21$

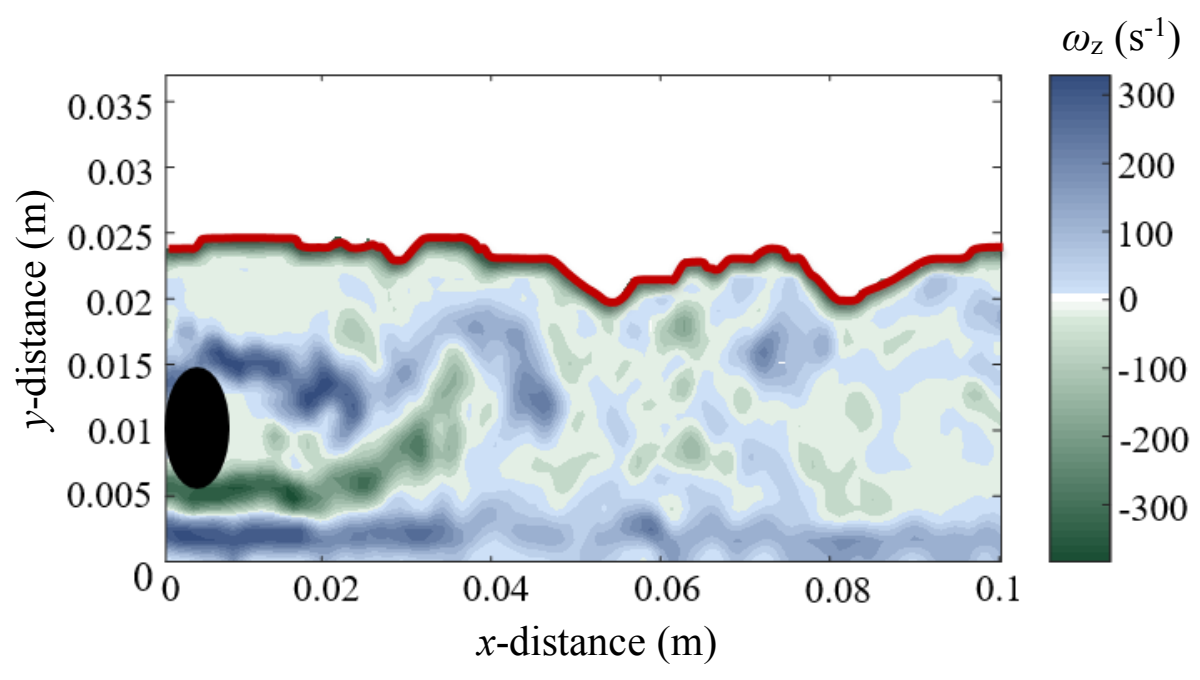

(b) $F r=1.86$

Figure 13 Vorticity field in the water phase of oil-water flows downstream of the $8 \mathrm{~mm}$ cylinder at (a) $Q_{\mathrm{o}}=10 \mathrm{~L} / \mathrm{min}$ and $Q_{\mathrm{w}}=10 \mathrm{~L} / \mathrm{min}$ (b) $Q_{\mathrm{o}}=10 \mathrm{~L} / \mathrm{min}$ and $Q_{\mathrm{w}}=20 \mathrm{~L} / \mathrm{min}$. The interface is shown as red line.

In the case of stratified liquid-liquid flows in a pipe, the interface height depends in a non-linear way on the flow rates of the two phases. As a result, the interface height and the in-situ water velocity, or the depth of the cylinder submergence and the Froude number based on water velocity, cannot be varied independently. Therefore, the effects of submergence depth and of Froude number on the shape of the interface could not be studied alone. Two cases with small change in $\mathrm{Fr}$ (difference in water velocity is 0.15 $\mathrm{m} / \mathrm{s}$ ) are selected to demonstrate the effects of submergence depth on the shape of the 
interface. The deformations of the interface in the wake of the $8 \mathrm{~mm}$ diameter cylinder at different submergence depths are given in Figure 13. As the submergence depth is increased (from $4 \mathrm{~mm}$ to $8 \mathrm{~mm}$ ) with increasing water flowrate from $10 \mathrm{~L} / \mathrm{min}$ to 20 $\mathrm{L} / \mathrm{min}$ the amplitude of the interfacial waves decreases. This is despite the increase in $F r$. The increase in $F r$ with the small increase in water velocity results in only a small increase of the vorticity magnitude while the area that the vortex occupies remains almost the same; this is in agreement with the results in single phase flow in Figures 7 and 8. The influence of $\mathrm{Fr}$ and submergence depth on the amplitude of interfacial waves will be analyzed further in the following sections based on the results from the high speed visualization.

\subsubsection{Frequency of vortex shedding and of interfacial waves}

The frequency of interfacial waves generated downstream of the cylinder was determined by measuring the number of wave crests passing through a fixed location over a period of time. Measurements were taken at location '2' in Figure 11, as the wave crest starts to form between locations ' 1 ' and ' 2 '. About 40 to 50 waves were considered for each case; these samples sizes gave averages that deviated by less than $5 \%$ from larger sample sizes. The Strouhal number was then calculated based on the wave frequency and the actual water velocity approaching the cylinder, estimated from the area occupied by the water and the water flowrate. For the $2 \mathrm{~mm}$ cylinder system, the flow conditions to determine the frequency based on high-speed visualization are limited to high oil to water ratios as the cylinder generates very small waves which cannot be easily tracked and analyzed. The frequency of vortex shedding in the water phase was determined from the velocity fields acquired from the PIV measurements as discussed in the single-phase flows section.

The frequencies of the vortices and of the interfacial waves generated behind the $2 \mathrm{~mm}$ and $8 \mathrm{~mm}$ cylinders at various mixture velocities are tabulated in Tables 5 and 6 respectively and plotted in Figure 14. For the two-phase flows, the Reynolds numbers based on the cylinder diameter, $R e_{D}$, and on the pipe diameter, $R e_{\text {pipe }}$ have been computed. based on water phase velocity, $u_{w}$ which is the phase where the cylinder is located. The $u_{w}$ is calculated based on the water flowrate, $Q_{w}$ and the area occupied by 
water phase in the pipe. In the tables, the corresponding $S t$ are also shown. For the 2 mm cylinder, the wave frequency increased significantly with $u_{\mathrm{w}}$ while for the $8 \mathrm{~mm}$ cylinder the increase was not as pronounced. With the $2 \mathrm{~mm}$ and the $8 \mathrm{~mm}$ cylinders used in the current work a large range of vortex shedding frequencies is generated. In all cases, the vortex shedding frequencies match the wave frequencies fairly well. This implies that the formation of waves at the interface of the stratified oil-water flows is induced by the vortices generated behind the cylinders submerged in water. The average $S t$ for the $2 \mathrm{~mm}$ cylinder system are in agreement with the literature values for unbounded flow. However, for the $8 \mathrm{~mm}$ cylinder cases the $S t$ are larger. The small gap between the wall and the $8 \mathrm{~mm}$ cylinder, which influences the vortex shedding frequency, also affects the interfacial waves which have lower frequency than those produced behind the $2 \mathrm{~mm}$ cylinder systems.

Table 5: Frequency and $S t$ of vortex shedding in water phase in the wake of the $2 \mathrm{~mm}$ and 8 mm cylinders

\begin{tabular}{cccccccc}
\hline $\begin{array}{c}D \\
(\mathrm{~mm})\end{array}$ & $\begin{array}{c}u_{\text {mix }} \\
(\mathrm{m} / \mathrm{s})\end{array}$ & $\begin{array}{c}r \\
(-)\end{array}$ & $\begin{array}{c}u_{\mathrm{w}} \\
(\mathrm{m} / \mathrm{s})\end{array}$ & $\begin{array}{c}R e_{D} \\
(-)\end{array}$ & $\begin{array}{c}R e_{\text {pipe }} \\
(-)\end{array}$ & $\begin{array}{c}f_{v} \\
(\mathrm{~Hz})\end{array}$ & $\begin{array}{c}S t \\
(-)\end{array}$ \\
\hline \multirow{6}{*}{2} & 0.310 & 1.00 & 0.342 & 684 & 12654 & 34.53 & 0.20 \\
& 0.465 & 2.00 & 0.414 & 828 & 15318 & 46.04 & 0.22 \\
& 0.620 & 1.00 & 0.645 & 1290 & 23865 & 62.06 & 0.20 \\
& 0.697 & 1.25 & 0.705 & 1410 & 26085 & 70.07 & 0.20 \\
& 0.774 & 1.50 & 0.762 & 1524 & 28194 & 75.08 & 0.20 \\
& 0.929 & 1.00 & 0.966 & 1932 & 35742 & 92.60 & 0.20 \\
& & & & & & & \\
& 0.310 & 1.00 & 0.342 & 2736 & 12654 & 10.50 & 0.25 \\
& 0.465 & 0.50 & 0.520 & 4160 & 19240 & 16.03 & 0.25 \\
& 0.620 & 1.00 & 0.645 & 5160 & 23865 & 22.05 & 0.27 \\
& 0.697 & 1.25 & 0.705 & 5640 & 26085 & 24.02 & 0.27 \\
& 0.774 & 1.50 & 0.762 & 6096 & 28194 & 24.32 & 0.27 \\
\hline
\end{tabular}


Table 6: Frequency and St of interfacial waves downstream of the $2 \mathrm{~mm}$ and $8 \mathrm{~mm}$ cylinders

\begin{tabular}{cccccccc}
\hline $\begin{array}{c}D \\
(\mathrm{~mm})\end{array}$ & $\begin{array}{c}u_{\mathrm{mix}} \\
(\mathrm{m} / \mathrm{s})\end{array}$ & $\begin{array}{c}r \\
(-)\end{array}$ & $\begin{array}{c}u_{\mathrm{w}} \\
(\mathrm{m} / \mathrm{s})\end{array}$ & $\begin{array}{c}R e_{D} \\
(-)\end{array}$ & $\begin{array}{c}R e_{\text {pipe }} \\
(-)\end{array}$ & $\begin{array}{c}f \\
(\mathrm{~Hz})\end{array}$ & $\begin{array}{c}S t \\
(-)\end{array}$ \\
\hline \multirow{4}{*}{2} & 0.465 & 2.00 & 0.415 & 830 & 15355 & 45.74 & 0.22 \\
& 0.620 & 3.00 & 0.539 & 1078 & 19943 & 60.30 & 0.22 \\
& 1.239 & 3.00 & 1.078 & 2156 & 39886 & 123.79 & 0.22 \\
& 0.310 & 1.00 & 0.342 & 2736 & 12654 & 11.30 & 0.26 \\
& 0.465 & 0.50 & 0.520 & 4160 & 19240 & 17.25 & 0.27 \\
& 0.620 & 1.00 & 0.645 & 5160 & 23865 & 21.52 & 0.27 \\
& 0.774 & 1.50 & 0.762 & 6096 & 28194 & 25.85 & 0.27 \\
& 0.929 & 2.00 & 0.872 & 6976 & 32264 & 29.60 & 0.27 \\
\hline
\end{tabular}

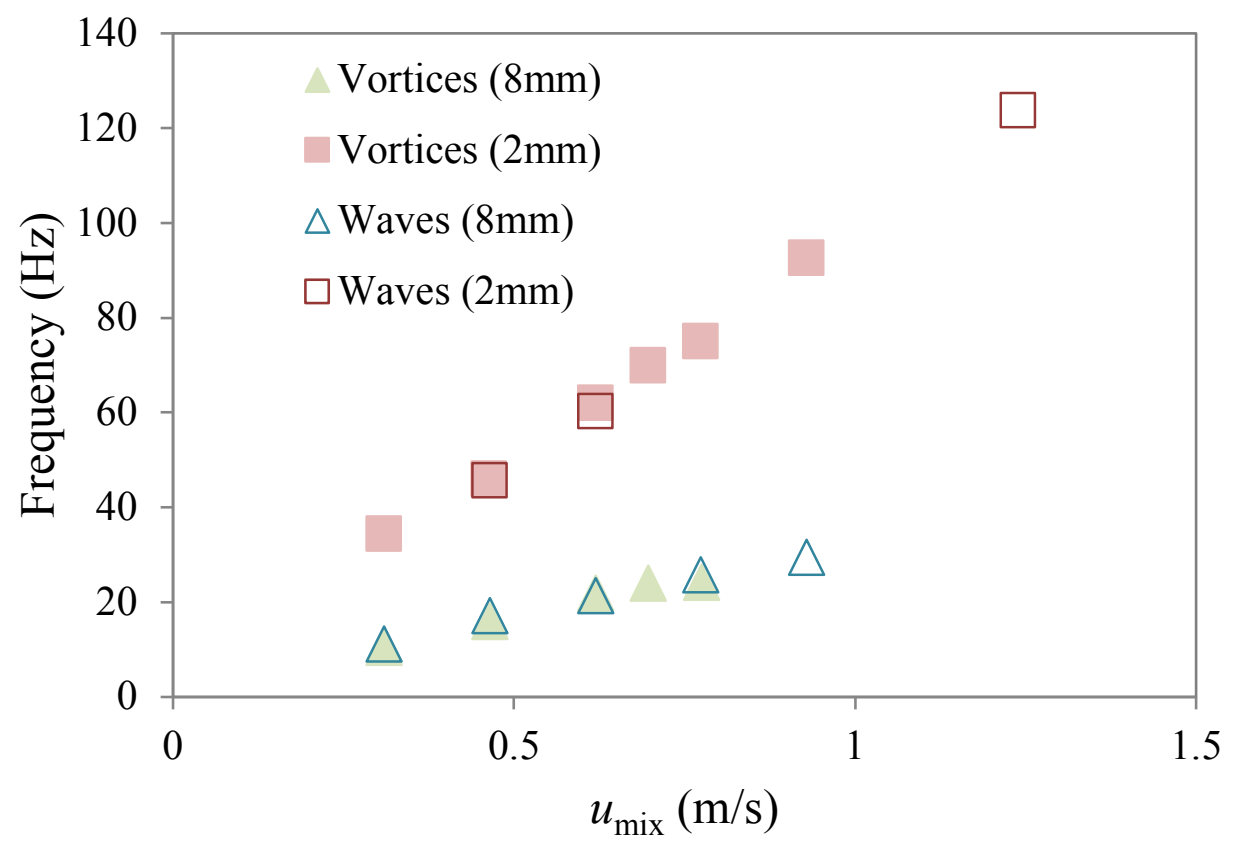

Figure 14: Frequency of interfacial waves and of von Karman vortices in the water phase of oil-water flows, downstream of cylinders with different sizes

\subsubsection{Amplitude of interfacial waves}

The amplitude of the interfacial waves is one of the important parameters related to the transition from separated to dispersed flows [4-5]. Above a critical wave amplitude, the waves will grow and drops may detach from their crests leading to dispersed flows. From the high-speed images, the average wave amplitude was determined by substracting the average interface height from the average wave crest height at each 
measuring location shown in Figure 11. The average interface height was calculated by measuring the interface height of the wave passing through a measuring location for $0.5 \mathrm{~s}$. Similarly, the average crest height was calculated by averaging the crest heights passing at each measuring location over $0.5 \mathrm{~s}$. The conditions studied are summarized in Table 7. However, results only for the $8 \mathrm{~mm}$ cylinder system will be discussed. This is because for the flow conditions investigated, the interfacial waves downstream of the $2 \mathrm{~mm}$ cylinder are very small and cannot be easily characterized. It should be noted that the experimental interface height is varied by the oil to water ratio, $r$; higher ratio leads to lower interface height and low submergence level of the cylinder.

Table 7: Froude number and incoming interface height for all flow conditions studied.

\begin{tabular}{|c|c|c|c|c|c|c|c|c|c|c|}
\hline \multicolumn{2}{|c|}{$Q(\mathrm{~L} / \mathrm{min})$} & \multirow{2}{*}{$\begin{array}{c}u_{\text {mix }} \\
(\mathrm{m} / \mathrm{s})\end{array}$} & \multirow{2}{*}{$\begin{array}{l}r \\
(-)\end{array}$} & \multirow{2}{*}{$\begin{array}{c}u_{\mathrm{w}} \\
(\mathrm{m} / \mathrm{s})\end{array}$} & \multirow[b]{2}{*}{$R e_{\text {pipe }}$} & \multicolumn{2}{|c|}{$\operatorname{Re}_{D}$} & \multicolumn{2}{|c|}{$F r$} & \multirow{2}{*}{$\begin{array}{c}\text { Interface } \\
\text { Height } \\
\text { (m) }\end{array}$} \\
\hline Oil & Water & & & & & $\begin{array}{c}2 \\
\mathrm{~mm}\end{array}$ & $\begin{array}{c}8 \\
\mathrm{~mm}\end{array}$ & $\begin{array}{c}2 \\
\mathrm{~mm}\end{array}$ & $\begin{array}{c}8 \\
\mathrm{~mm}\end{array}$ & \\
\hline 10 & 10 & 0.310 & 1.00 & 0.342 & 12580 & 680 & 2720 & 2.43 & 1.21 & 0.017 \\
\hline 10 & 20 & 0.465 & 0.50 & 0.520 & 19240 & 1040 & 4160 & 3.71 & 1.86 & 0.021 \\
\hline 20 & 20 & 0.629 & 1.00 & 0.645 & 24050 & 1300 & 5200 & 4.64 & 2.32 & 0.017 \\
\hline 20 & 30 & 0.774 & 0.67 & 0.825 & 30710 & 1660 & 6640 & 5.94 & 2.97 & 0.020 \\
\hline 30 & 20 & 0.774 & 1.50 & 0.762 & 28120 & 1520 & 6080 & 5.43 & 2.71 & 0.016 \\
\hline 30 & 30 & 0.929 & 1.00 & 0.965 & 35890 & 1940 & 7760 & 6.93 & 3.46 & 0.017 \\
\hline 30 & 40 & 1.085 & 0.75 & 1.155 & 42920 & 2320 & 9280 & 8.28 & 4.14 & 0.019 \\
\hline 40 & 30 & 1.085 & 1.33 & 1.087 & 40330 & 2180 & 8720 & 7.78 & 3.89 & 0.013 \\
\hline 40 & 40 & 1.239 & 1.00 & 1.285 & 47730 & 2580 & 10320 & 9.21 & 4.60 & 0.013 \\
\hline
\end{tabular}

The average wave amplitudes at constant flowrate ratio $(r=1)$ and different $F r$ are presented in Figure 15 for the $8 \mathrm{~mm}$ cylinder. At these flow conditions, the incoming interface height and the corresponding submergence depth are constant. Thus, an increase in $\mathrm{Fr}$ is related to an increase in water velocity as well as mixture velocity. As can be seen, the wave amplitude increases downstream of the cylinder up to a maximum value before it reduces again. As the velocity increases, the magnitude of the von Karman vortices shed also increases which affects the amplitude of the interfacial waves. Also, the vortices which are attached on the waves expand with distance from the cylinder. At a certain distance from the cylinder, though, the vortices start to decay. There is limited work reported on the far wake region behind a bluff body, and Williamson [12] in a review reported that the von Karman street decays downstream of the bluff body. 


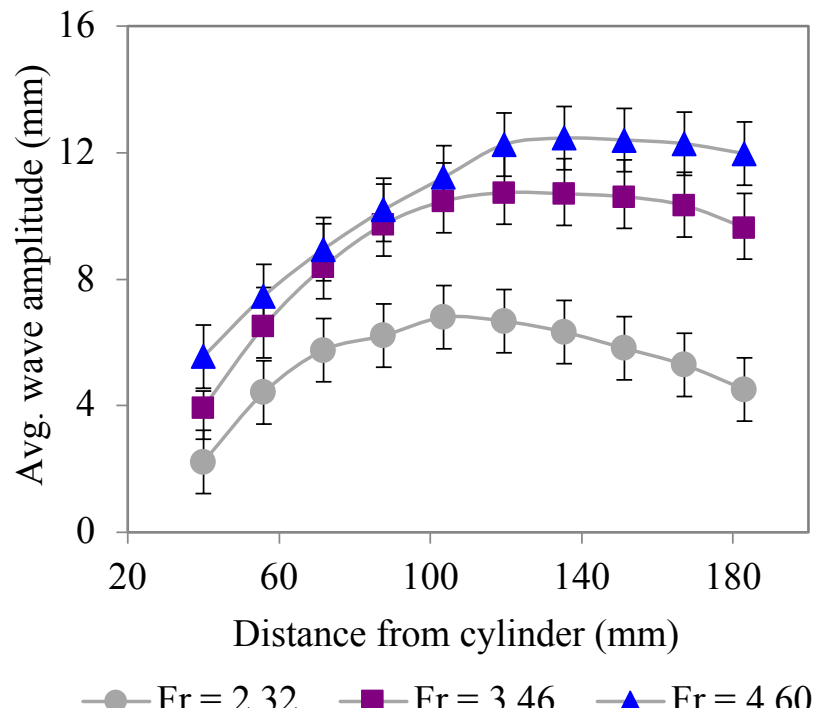

Figure 15: Effect of Froude number on interfacial wave amplitudes $(D=8 \mathrm{~mm}, r=1)$

To support this, an example of a CFD simulation of single-phase flow is shown in Figure 16 for $u=0.645 \mathrm{~m} / \mathrm{s}$ which corresponds to $F r=2.32$. It can be seen that as the vortices are convected downstream the flow, the area covered by the vortices and the vorticity magnitude start to decline at a certain distance behind the cylinder. The decay of the vortices results in a reduction of the wave amplitude. This change happens further downstream of the cylinder as the velocity increases because at high velocities the vortices and the waves are convected over a longer distance downstream. It should be noted, however, that although the vortices and waves generated by the cylinder decay downstream, the drops that form close to the bluff body can be sustained for a long distance and give rise to dual continuous patterns.
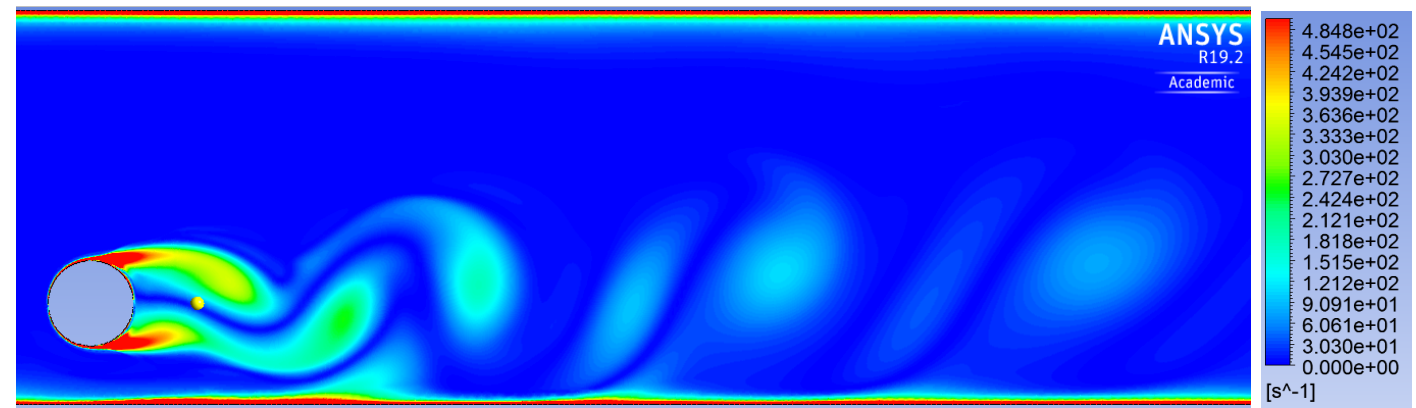

Figure 16: Instantaneous vorticity field in the wake of the $8 \mathrm{~mm}$ cylinder for single phase water flow at $u=0.645 \mathrm{~m} / \mathrm{s}$ obtained from CFD 
The effect of the incoming interface height on interfacial wave amplitude at constant $u_{\mathrm{mix}}=0.774 \mathrm{~m} / \mathrm{s}$ is shown in Figure 17. The interface height is controlled by the oil-towater ratio; higher ratio leads to lower interface height and low submergence level of the cylinder (see Table 7). When the interface height is low, the von Karman vortices have a more significant impact on the interface as discussed for Figure 13. As a result, waves with large amplitudes are generated. The wave amplitudes generated with the 8 $\mathrm{mm}$ cylinder are also compared against those generated by a $5 \mathrm{~mm}$ cylinder measured by Park et al. [6] in the same flow system, in Figure 18. For both cylinder sizes the wave amplitudes increase with distance from the cylinder. The large $8 \mathrm{~mm}$ cylinder produces waves with larger amplitude compared to the $5 \mathrm{~mm}$ cylinder at the same flow conditions. This is attributed to the size of the vortices shed by the different cylinders. As was shown in Figure 6, the size of the vortices increases with the cylinder diameter.

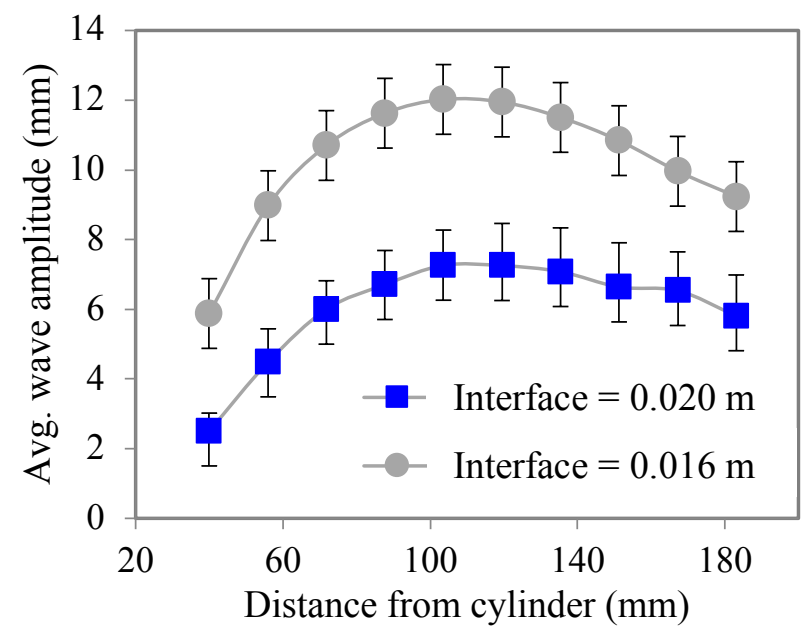

Figure 17: Effects of incoming interface height on interfacial wave amplitudes at $u_{\text {mix }}=0.774$ $\mathrm{m} / \mathrm{s}$ for flowrates of $\mathrm{Q}_{\text {oil }}=20 \mathrm{~L} / \mathrm{min}$ and $\mathrm{Q}_{\mathrm{w}}=30 \mathrm{~L} / \mathrm{min}$ (Interface height $=20 \mathrm{~mm}$ ) and $\mathrm{Q}_{\text {oil }}=$ $30 \mathrm{~L} / \mathrm{min}$ and $\mathrm{Q}_{\mathrm{w}}=20 \mathrm{~L} / \mathrm{min}($ Interface height $=16 \mathrm{~mm}$ ) for a system with cylinder $D=8 \mathrm{~mm}$ 


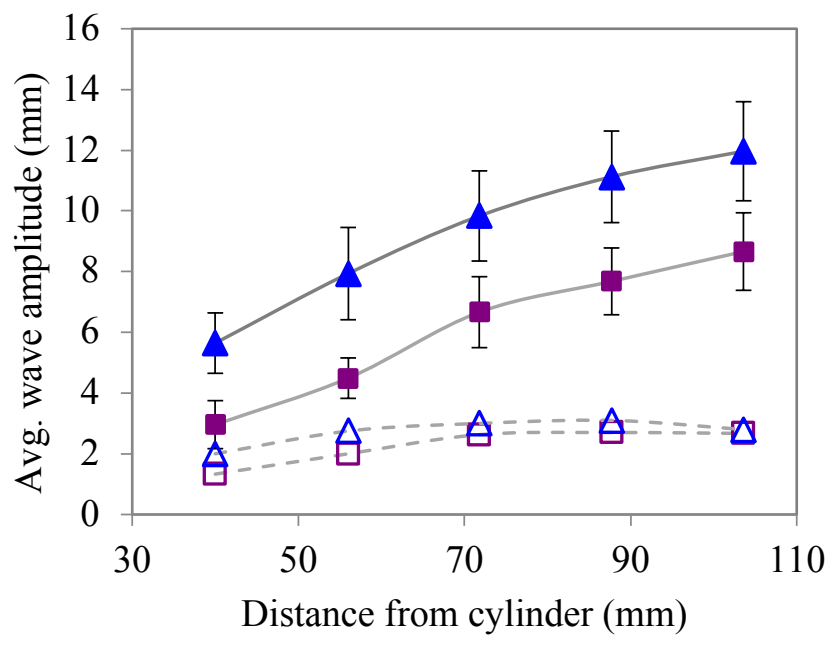

$\square 8 \mathrm{~mm}, \mathrm{r}=0.75-\square-5 \mathrm{~mm}, \mathrm{r}=0.75$
$\square-8 \mathrm{~mm}, \mathrm{r}=1.33-\triangle-5 \mathrm{~mm}, \mathrm{r}=1.33$

Figure 18: Effects of flowrate ratio on wave amplitude for different cylinder sizes for $u_{\text {mix }}=1.085 \mathrm{~m} / \mathrm{s}$ for flowrates of $\mathrm{Q}_{\text {oil }}=30 \mathrm{~L} / \mathrm{min}$ and $\mathrm{Q}_{\mathrm{w}}=40 \mathrm{~L} / \mathrm{min}(r=0.75)$ and $\mathrm{Q}_{\text {oil }}=$ $40 \mathrm{~L} / \mathrm{min}$ and $\mathrm{Q}_{\mathrm{w}}=30 \mathrm{~L} / \mathrm{min}(r=1.33)$

\subsection{Conclusions}

Cylindrical bluff bodies of various sizes were used to induce interfacial waves in twophase, oil-water flows and to promote the transition from separated to dispersed patterns. The waves resulted from the interaction of the von Karman vortices shed by the cylinders with the oil-water interface in stratified flows. Computational Fluid Dynamics simulations were used to select the bluff body diameters that would shed vortices with frequencies spanning two orders of magnitude. The simulations were twodimensional but agreed well with experimental velocity field data taken in the middle plane of the pipe, which demonstrates that fast 2D simulations can be used to estimate the flow properties behind a bluff body in pipes.

The selected cylinder diameters, $2 \mathrm{~mm}$ and $8 \mathrm{~mm}$, produced vortices with frequencies between 1 to $100 \mathrm{~Hz}$ in horizontal oil-water pipe flows. High speed imaging was used to acquire the flow patterns and the interfacial wave characteristics while Particle Image Velocimetry was used to obtain velocity fields and vortex properties behind the 
cylinders. The experimental data in single phase water flow showed that the $2 \mathrm{~mm}$ cylinder produced vortices with Strouhal numbers that matched the literature values for unbounded flows, while the $8 \mathrm{~mm}$ cylinder resulted in vortices with higher Strouhal numbers. This increase was attributed to the short distance between the cylinder and the bottom pipe wall.

For all two-phase flow cases studied, the frequencies of the vortices and the corresponding Strouhal numbers matched the frequencies of the vortex induced interfacial waves. This suggests that changes in the vortex shedding properties with flowrates or with bluff body size are reflected on the interfacial wave characteristics. It is possible, therefore, to control the properties of the interfacial waves in oil-water flows through the bluff body design. The transition boundary from stratified to dispersed flows shifted towards lower mixture velocities when cylinders were present in the water phase and was affected by the cylinder size. It was found that the large, 8 $\mathrm{mm}$, cylinder generated interfacial waves with larger amplitude compared to the $2 \mathrm{~mm}$ cylinder, and caused an ealier transition to dispersed patterns.

In the future, different shapes of the bluff body and their effects on the vortices and on the interfacial waves will be investigated. By using fluids with matching refractive index, it is possible to obtain measurements close to the interface for very wavy interfaces and when drops detach. Ths will extend the range of flowrates than can be investigated with PIV and reveal the mechanisms of drop detachment from the interfacial waves.

\section{Acknowledgments}

The project was supported by the EPSRC Programme Grant MEMPHIS. K.H Park would like to thank UCL for his studentship and M. Zainal Abidin would like to thank the University of Malaya for his studentship. 


\subsection{References}

[1] J.L. Trallero, C. Sarica, J.P. Brill, A study of oil - water flow patterns in horizontal pipes, SPE Production and Facilities 12 (1997) 165-172.

[2] J. Lovick and P. Angeli, Droplet size and velocity profiles in liquid-liquid horizontal flows, Chemical Engineering Science 59 (2004) 3105-3115.

[3] A. Al-Sarkhi, E. Pereyra, I. Mantilla, C. Avila, Dimensionless oil-water stratified to non-stratified flow pattern transition, Journal of Petroleum Science and Engineering 151 (2017) 284-291.

[4] T. Al-Wahaibi, P. Angeli, Transition between stratified and non-stratified horizontal oil-water flows. Part I: Stability analysis, Chemical Engineering Science 62 (2007) 2915-2928.

[5] T. Al-Wahaibi, P. Angeli, Experimental study on interfacial waves in stratified horizontal oil-water flow, International Journal of Multiphase Flow 37 (2011) 930-940.

[6] K.H. Park, M. Chinaud, P. Angeli,Transition from stratified to non-stratified oil - water flows using a bluff body, Experimental Thermal and Fluids Science 76 (2016) 175-184.

[7] M. Chinaud, K.H. Park, P. Angeli, Flow pattern transition in liquid-liquid flows with a transverse cylinder, International Journal of Multiphase Flow 90 (2017) 1-12.

[8] A. Murakami, S. Mochida, On turbulent vortex shedding flow past 2D square cylinder predicted by CFD, Journal of Wind Engineering and Industrial Aerodynamics 55 (1995) 191-211.

[9] L. Bruno, D. Fransos, N. Coste, A. Bosco, 3D flow around a rectangular cylinder : A computational study, Journal of Wind Engineering and Industrial Aerodynamics 98 (2008) 20-24.

[10] P.P. Patil, S. Tiwari, Effect of blockage ratio on wake transition for flow past square cylinder, Fluid Dynamics Research 40 (2008),. 753-778.

[11] I. Kumagai, Y. Murai, Y. Tasaka,Science and bubble generation by a cylinder moving beneath a free surface, Journal of Fluid Science and Technology 6 (2011) 851-859.

[12] C.H.K. Williamson, Vortex dynamics in the cylinder wake, Annual Review of Fluid Mechanics 28 (1996) 477-539.

[13] L. Zovatto, G. Pedrizzetti, Flow about a circular cylinder between parallel walls, Journal of FLuid Mechanics 440 (2001) 1-25. 
[14] F. Rehimi, F. Aloui, S. B. Nasrallah, L. Doubliez, J. Legrand, Experimental investigation of a confined flow downstream of a circular cylinder centred between two parallel walls, Journal of Fluids and Structures 24 (2008) 855882.

[15] C. Lei, L. Cheng, K. Kavanagh, Re-examination of the effect of a plane boundary on force and vortex shedding of a circular cylinder, Journal of Wind Engineering and Industrial Aerodynamcis 80 (1999) 263-286.

[16] S.J. Price, D. Sumner, J.G. Smith, K. Leong, M.P. Paidoussis, Initial pressure distribution due to jet impact on a rigid body, Journal of Fluids and Structure 16 (2002) 175-191.

[17] A. Dipankar, T.K. Sengupta, Flow past a circular cylinder in the vicinity of a plane wall, Journal of Fluids and Structures 20 (2005) 403-423.

[18] P. Reichl, K. Hourigan, M.C. Thompson, Flow past a cylinder close to a free surface, Journal of Fluid Mechanics 533 (2005) 269-296.

[19] M.Y. Lin, L.H. Huang, Free-surface flow past a submerged cylinder, Journal of Hydrodynamics 22 (2010) 209-214.

[20] J. Sheridan, J.C. Lin, D. Rockwell, Flow past a cylinder close to a free surface, Journal of Fluid Mechanics 330 (1997) 1-30.

[21] Z. Huang, J.A. Olson, R.J. Kerekes, S.I. Green, Numerical simulation of the flow around rows of cylinders, Computers and Fluids 35 (2006) 485-491.

[22] H. Bai, J. Li, Numerical simulation of flow over a circular cylinder at low reynolds number, Advance Materials Research 225-260 (2011) 942-946.

[23] M. Rahman, M. Karim, A. Alim, Numerical investigation of unsteady flow past a circular cylinder using 2-d finite volume method, Journal of Naval Architecture and Marine Engineering 4 (2007) 27-42.

[24] M. Chen, A. Kamath, H. Bihs, M. Saud, Numerical simulation of free-surface waves past two semi- submerged horizontal circular cylinders in tandem, Marine Structures 52 (2017) 1-14.

[25] H. Liang, Z. Zong, L. Zou, L. Zhou, L. Sun, Vortex shedding from a twodimensional cylinder beneath a rigid wall and a free surface according to the discrete vortex method, European Journal of Mechanics B/Fluids 43 (2014) $110-119$.

[26] B. Chen, L. Lu, C.A. Greated, H. Kang, Investigation of wave forces on partially submerged horizontal cylinders by numerical simulation, Ocean Engineering 107 (2015) 23-31.

[27] C. Bozkaya, S. Kocabiyik, Free surface wave interaction with an oscillating cylinder, Applied Mathematics Letters 27 (2014) 79-84. 
[28] J. Shao, C. Zhang, Numerical analysis of the flow around a circular cylinder using RANS and LES, International Journal of Computational Fluid Dynamics 20 (2006) 301-307.

[29] M. Breuer, Large eddy simulation of the sub-critical flow past a circular cylinder: Numerical and modeling aspects, International Journal for Numerical Methods in Fluids 28 (1998) 1281-1302.

[30] D. Rocchi, A. Zasso, Vortex shedding from a circular cylinder in a smooth and wired configuration: Comparison between 3D LES simulation and experimental analysis, Journal of Wind Engineering and Industrial Aerodynamics 90 (2002) 475-489.

[31] X.K. Wang, S.K. Tan, Comparison of flow patterns in the near wake of a circular cylinder and a square cylinder placed near a plane wall, Ocean Engineering 35 (2008) 458-472.

[32] P. Catalano, M. Wang, G. Iaccarino, P. Moin, Numerical simulation of the flow around a circular cylinder at high Reynolds numbers, International Journal of Heat and Fluid Flow 24 (2003) 463-469.

[33] Q.H. Mazumder, CFD analysis of single and multiphase flow characteristics in elbow, Engineering 4 (2012) 210-214.

[34] A. Renfer, M.K. Tiwari, F. Meyer, T. Brunschwiler, B. Michel, D. Poulikakos, Vortex shedding from confined micropin arrays, Macrofluidics and Nanofluidics 15 (2013) 231-242.

[35] A.H. Barral, P. Angeli, Interfacial charateristics of stratified liquid-liquid flows using conductance probe, Experiments in Fluids 54 (2013) 1604-1618. 
Nomenclatures

\begin{tabular}{ccc}
\hline Symbols & Description & Unit \\
\hline$R e_{D}$ & Cylinder diameter based Reynolds number & {$[-]$} \\
$u_{\text {mix }}$ & Mixture velocity & {$[\mathrm{m} / \mathrm{s}]$} \\
$D$ & Cylinder diameter & {$[\mathrm{m}]$} \\
$S t$ & Strouhal number & {$[-]$} \\
$f$ & Frequency & {$[\mathrm{Hz}]$} \\
$f_{v}$ & Vortex shedding frequency & {$[\mathrm{Hz}]$} \\
$F r$ & Froude number & {$[-]$} \\
$g$ & Gravitational acceleration & {$\left[\mathrm{m} / \mathrm{s}^{2}\right]$} \\
$h_{s}$ & Submergence depth & {$[\mathrm{m}]$} \\
$h$ & Distance from cylinder to wall & {$[\mathrm{m}]$} \\
$Q$ & Flowrate & {$[\mathrm{L} / \mathrm{min}]$} \\
$Q_{o}$ & Oil input flowrate & {$[\mathrm{L} / \mathrm{min}]$} \\
$Q_{w}$ & Water input flowrate & {$[\mathrm{L} / \mathrm{min}]$} \\
$R$ & Pipe radius & {$[\mathrm{m}]$} \\
$r$ & Oil to water flowrate ratio & {$[-]$} \\
$t$ & time & {$[\mathrm{s}]$} \\
$u$ & Velocity & {$[\mathrm{m} / \mathrm{s}]$} \\
\hline
\end{tabular}

\begin{tabular}{ccc}
\hline $\begin{array}{c}\text { Greek } \\
\text { letters }\end{array}$ & Description & Unit \\
\hline$\rho$ & Density & {$\left[\mathrm{Kg} / \mathrm{m}^{3]}\right.$} \\
$\mu$ & Viscosity & {$[\mathrm{Pa} \mathrm{s}]$} \\
$r$ & Gap ratio & {$[-]$} \\
$\omega_{z}$ & Spanwise vorticity & {$\left[\mathrm{s}^{-1}\right]$} \\
\hline
\end{tabular}

